\title{
REDUCTION AND LIFTING OF SPECIAL METACYCLIC COVERS
}

\author{
BY STEFAN WEWERS
}

ABSTRACT. - Special covers are metacyclic covers of the projective line, with Galois group $\mathbb{Z} / p \rtimes \mathbb{Z} / m$, which have a specific type of bad reduction to characteristic $p$. Such covers arise in the study of the arithmetic of Galois covers of $\mathbb{P}^{1}$ with three branch points. Our results provide a simple description of special covers in terms of certain lifting data in characteristic $p$.

(C) 2003 Éditions scientifiques et médicales Elsevier SAS

RÉSUMÉ. - On définit les revêtements spéciaux comme des revêtements métacycliques de la droite projective ayant un certain type de mauvaise réduction. Ces revêtements apparaissent naturellement dans l'étude des revêtements galoisiens étales de la droite projective moins trois points. Nos résultats donnent une description simple des revêtements spéciaux comme relèvements de certaines données en caractéristique $p$.

(C) 2003 Éditions scientifiques et médicales Elsevier SAS

\section{Introduction}

In [13] Raynaud has given a criterion for good reduction of Galois covers of the projective line which are ramified at three points. The proof of this criterion depends on the analysis of Galois covers with bad reduction, under certain conditions on the Galois group. In one particular step of this analysis, Raynaud introduced the notion of the auxiliary cover: to a $G$-Galois cover $f: Y \rightarrow \mathbb{P}^{1}$ with bad reduction to characteristic $p$ he associates (under certain conditions) an $H$-Galois cover $f_{\text {aux }}: Y_{\text {aux }} \rightarrow \mathbb{P}^{1}$, which has, in some sense, the same type of bad reduction as $f$, but whose Galois group $H$ is a certain solvable quotient of a subgroup of $G$. For instance, if $p$ strictly divides the order of $G, H$ is a metacyclic group, isomorphic to $\mathbb{Z} / p \rtimes \mathbb{Z} / m$. Thus, for many purposes, the study of bad reduction of Galois covers can be reduced to the study of covers with certain solvable (in the easiest case, metacyclic) Galois groups. However, this reduction step is paid for by the introduction of extra branch points (the branch locus of $f$ is a subset of the branch locus of $f_{\text {aux }}$ ). In general, it is hard to predict where these extra branch points occur.

The present paper is concerned with a detailed study of metacyclic covers of $\mathbb{P}^{1}$, with Galois group isomorphic to $\mathbb{Z} / p \rtimes \mathbb{Z} / m$, which arise as the auxiliary cover of $G$-covers of $\mathbb{P}^{1}$ with three branch points and prime-to- $p$ ramification. In Section 1, we give a characterization of such covers which is independent of the group $G$; this characterization gives rise to the definition of special metacyclic covers. In Section 2 we show that the reduction of a special cover to characteristic $p$ is as simple as one can expect it to be; in particular, it is determined by a so-called special degeneration datum. Finally, in Section 3 we show that one can lift any special degeneration datum to a special cover. In a subsequent paper [21], we will strengthen this lifting result. Also, 
in [20] we will apply our results on special covers to the study of three point covers with bad reduction.

We shall now give a more detailed outline of our results. Fix a prime number $p$ and a finite group $G$ such that $p$ strictly divides the order of $G$. Let us also fix a field $K_{0}$ of characteristic 0 which is complete with respect to a discrete valuation $v$. We assume that the residue field $k$ of $v$ is algebraically closed and of characteristic $p$. Suppose we are given a $G$-Galois cover $f: Y \rightarrow \mathbb{P}^{1}$ defined over a finite extension $K / K_{0}$, branched at $x_{1}=0, x_{2}=1$ and $x_{3}=\infty$, with primeto- $p$ ramification. We assume that $f$ has bad reduction at $v$, and has a stable model over the ring of integers of $K$ (the latter holds after replacing $K$ by a finite extension). Let $\bar{f}: \bar{Y} \rightarrow \bar{X}$ be the stable reduction of $f$. For a precise definition of the stable reduction, see Section 1.3. Let us only say that $\bar{f}$ is a finite $G$-invariant map between semistable curves over $k$ which is inseparable over certain irreducible components of $\bar{X}$. By a result of Raynaud [13], the cover $\bar{f}$ is separable exactly over the tails of $\bar{X}$, i.e. those irreducible components that are connected to the rest of $\bar{X}$ in a single point. Moreover, the three branch points $x_{1}=0, x_{2}=1, x_{3}=\infty$ of $f$ specialize to points on pairwise distinct tails $\bar{X}_{1}, \bar{X}_{2}, \bar{X}_{3}$. Let $\bar{X}_{4}, \ldots, \bar{X}_{r}$ be the remaining tails, and let $\mathcal{D}_{i} \subset\left(\mathbb{P}_{K}^{1}\right)^{\text {rig }}$ be the closed rigid disk corresponding to the tail $\bar{X}_{i}$, for $i=1, \ldots, r$. By definition, $x_{i} \in \mathcal{D}_{i}$ for $i=1,2,3$.

Let us choose $K$-rational points $x_{i} \in \mathcal{D}_{i}$, for $i=4, \ldots, r$. After having made this choice, we can define the auxiliary cover $f_{\text {aux }}: Y_{\text {aux }} \rightarrow \mathbb{P}^{1}$ associated to $f$. This is a certain $H$-Galois cover, branched at $x_{1}, \ldots, x_{r}$, where $H$ is the quotient of a subgroup of $G$, isomorphic to $\mathbb{Z} / p \rtimes \mathbb{Z} / m$ for some integer $m>1$ dividing $p-1$. The cover $f_{\text {aux }}$ has bad reduction, as well, and its stable reduction $\bar{f}_{\text {aux }}: \bar{Y}_{\text {aux }} \rightarrow \bar{X}$ is closely related to the stable reduction of $f$ (for instance, $\bar{f}$ and $\bar{f}_{\text {aux }}$ have the same target $\bar{X}$ ). Of course, the cover $f_{\text {aux }}$ depends on the choice of the extra branch points. Raynaud's results, together with the fact that $f$ has only three branch points, impose very strong conditions on the auxiliary $f_{\text {aux }}$. Furthermore, it is demonstrated in [13] that the understanding of the arithmetic of $f$ can be reduced, to some extend, to the study of its auxiliary cover. This motivates the study of special covers.

So from now on, we let $f: Y \rightarrow \mathbb{P}^{1}$ denote an $H$-cover defined over $K$, where $K / K_{0}$ and $H=\mathbb{Z} / p \rtimes \mathbb{Z} / m$ are as before. We say that $f$ is special if it has bad reduction and its stable reduction $\bar{f}: \bar{Y} \rightarrow \bar{X}$ satisfies some rather restrictive conditions (which are satisfied by auxiliary covers of three point covers, as above). In particular, the branch points $x_{1}, \ldots, x_{r}$ of $f$ specialize to pairwise distinct tails $\bar{X}_{1}, \ldots, \bar{X}_{r}$ of $\bar{X}$. Our first main result is:

TheOREM A. - Let $f: Y \rightarrow \mathbb{P}^{1}$ be a special $H$-cover over $K$ and $\bar{f}: \bar{Y} \rightarrow \bar{X}$ its stable reduction. Then the (semistable) curve $\bar{X}$ is the union of $r+1$ distinct irreducible components $\bar{X}_{0}, \ldots, \bar{X}_{r}$ (each isomorphic to $\mathbb{P}_{k}^{1}$ ) such that, for $i=1, \ldots, r, \bar{x}_{i} \in \bar{X}_{i}$ and $\bar{X}_{i}$ meets $\bar{X}_{0}$ in a unique point $\tau_{i}$. In particular, $x_{i} \not \equiv x_{j} \bmod v$ for $i \neq j$ (as points on $\mathbb{P}^{1}$ ).

The essential content of Theorem A is that the stable reduction of a special cover is as simple as one can expect it to be. For instance, if $f: Y \rightarrow \mathbb{P}^{1}$ is the auxiliary cover of a three point $G$-cover $\tilde{f}: \tilde{Y} \rightarrow \mathbb{P}^{1}$, then Theorem A implies that the disks $\mathcal{D}_{1}, \ldots, \mathcal{D}_{r}$ determined by the stable reduction of $\tilde{f}$ are equidistant. Note that this is in general wrong for $G$-covers with more than three branch points.

The proof of Theorem A shows that the stable reduction of a special cover is determined by a pair $\left(\bar{Z}_{0}, \omega_{0}\right)$, where $\bar{Z}_{0} \rightarrow \mathbb{P}_{k}^{1}$ is an $m$-cyclic cover branched at the points $\tau_{1}, \ldots, \tau_{r}$ (as in Theorem A), and $\omega_{0}$ is a regular differential form on $\bar{Z}_{0}$. The differential form $\omega_{0}$ verifies the following conditions: a.) $\omega_{0}$ is logarithmic, i.e. of the form $\mathrm{d} u / u, \mathrm{~b}$.) $\omega_{0}$ is an eigenvector under the action of $\mathbb{Z} / m$ and c.) the zeros of $\omega_{0}$ are contained in the ramification locus of $\bar{Z}_{0} \rightarrow \mathbb{P}_{k}^{1}$. Let us call the pair $\left(\bar{Z}_{0}, \omega_{0}\right)$ a special degeneration datum. It turns out that condition $\mathrm{b}$.) and c.) already determine the differential $\omega_{0}$ up to a constant factor. Therefore, condition a.) poses a 
restriction on the cover $\bar{Z}_{0} \rightarrow \mathbb{P}_{k}^{1}$ and, in particular, on the branch points $\tau_{1}, \ldots, \tau_{r}$. In concrete terms, this condition translates into an explicit system of equations satisfied by the tuple $\left(\tau_{i}\right)$. One can show that this system has only a finite number of solutions, and that therefore there exist only a finite number of nonisomorphic special degeneration data, for a given prime $p$.

Our second main result is that every special degeneration datum arises from a special $H$-cover. More precisely, we have:

THEOREM B. - Let $\left(\bar{Z}_{0}, \omega_{0}\right)$ be a special degeneration datum, defined over an algebraically closed field $k$ of characteristic $p$. Let $K_{0}$ be the fraction field of the ring $W(k)$ of Witt vectors over $k$, and let $x_{1}, \ldots, x_{r}$ be $K_{0}$-rational points on $\mathbb{P}^{1}$ lifting the branch points of $\bar{Z}_{0} \rightarrow \mathbb{P}_{k}^{1}$. Then there exists a special $H$-cover $f: Y \rightarrow \mathbb{P}^{1}$ with branch points $x_{1}, \ldots, x_{r}$ whose reduction $\bar{f}: \bar{Y} \rightarrow \bar{X}$ corresponds to $\left(\bar{Z}_{0}, \omega_{0}\right)$. The cover $f$ is defined over the tame extension $K / K_{0}$ of degree $(p-1) / m$ and is uniquely determined by $\left(\bar{Z}_{0}, \omega_{0}\right)$ and $\left(x_{1}, \ldots, x_{r}\right)$.

Theorem $\mathrm{B}$ is a result on lifting inseparable covers of curves from characteristic $p$ to characteristic 0. In this sense, it is similar to the main result of [8]. But even though [8] has had a great influence on the present paper (this is most obvious in Section 2), the proof of Theorem B uses a very different approach. First, the results of [8] are formulated in terms of automorphisms of the $p$-adic open disk (and are therefore something local), whereas we are dealing with projective curves. Second, for the applications in [20] it is important to prove uniqueness of lifting and to have a good control over the field of definition. Since we are concerned with lifting inseparable covers, the usual strategy of first lifting locally and then using formal patching to obtain a global lifting breaks down.

Our proof of Theorem B is based on an analysis of the $p$-torsion of the Jacobian of the intermediate $m$-cyclic cover and depends crucially on the assumption that $K_{0}$ is absolutely unramified. It is divided into two steps. Let $K_{0}$ and $\left(\bar{Z}_{0}, \omega_{0}\right)$ be as in the statement of Theorem B. Moreover, let $K / K_{0}$ be a finite extension and $x_{1}, \ldots, x_{r} K$-rational points on $\mathbb{P}^{1}$ lifting the branch points of $\bar{Z}_{0} \rightarrow \mathbb{P}_{k}^{1}$. In the first step, we show that there exists a unique $H$-cover $f: Y \rightarrow \mathbb{P}^{1}$ with branch points $x_{1}, \ldots, x_{r}$, defined over a tame extension of $K$, whose stable reduction gives rise to the special deformation datum $\left(\bar{Z}_{0}, \omega_{0}\right)$. In the second step we show that $f$ is special if $K=K_{0}$. It is easy to see that the condition $K=K_{0}$ is not necessary for $f$ to be special. To see this, let $x_{1}, \ldots, x_{r}$ be $K_{0}$-rational and $f$ the corresponding lift. Since $f$ is special by Theorem $\mathrm{B}$, its stable reduction gives rise to closed rigid disks $\mathcal{D}_{1}, \ldots, \mathcal{D}_{r}$ such that $x_{i} \in \mathcal{D}_{i}$. One can show that the disks $\mathcal{D}_{i}$ do not depend on the choice of $x_{1}, \ldots, x_{r}$. Let $x_{i}^{\prime}$ be an arbitrary point in $\mathcal{D}_{i}$ (not necessarily $K_{0}$-rational) and let $f^{\prime}$ be the lift corresponding to the tuple $x_{1}^{\prime}, \ldots, x_{r}^{\prime}$. Then a formal patching argument shows that $f^{\prime}$ is special, as well.

We will show in [21], using an entirely different method, that $x_{i} \in \mathcal{D}_{i}$ is in fact a necessary condition for the lift $f$ to be special. This result has very nice consequences, see [20]. For instance, if the special cover $f$ is the auxiliary cover of a three point cover $\tilde{f}: \tilde{Y} \rightarrow \mathbb{P}^{1}$, then $\tilde{f}$ can be defined over a tame extension of $K_{0}$. Therefore, the field of moduli of a three point covers is at most tamely ramified at $p$ provided that $p^{2}$ does not divide the order of the monodromy group.

\section{Special metacyclic covers}

In this paper we consider metacyclic covers $f: Y \rightarrow \mathbb{P}^{1}$ which are the composition of a ramified $m$-cyclic cover $Z \rightarrow \mathbb{P}^{1}$ and an étale $p$-cyclic cover $Y \rightarrow Z$, with Galois group $\mathbb{Z} / p \rtimes \mathbb{Z} / m$. Such a cover corresponds to an element of a certain isotypical component of the $p$-torsion of the Jacobian $J_{Z}$. We give a numerical criterion which implies that this isotypical component has étale reduction. This forces the cover $Y \rightarrow Z$ to have bad (more specifically, 
multiplicative) reduction, and allows us to compute the field of moduli of the cover $f$, see Section 1.2. In Section 1.3, we define special covers as metacyclic covers $f: Y \rightarrow \mathbb{P}^{1}$ whose stable reduction satisfies certain (rather restrictive) conditions. In Section 1.4, we show that the auxiliary cover of a Galois cover of $\mathbb{P}^{1}$ branched at three points is special, provided that $p$ strictly divides the order of the Galois group.

\subsection{Metacyclic covers of multiplicative type}

Let us fix the following objects:

- A complete discrete valuation ring $R_{0}$, with fraction field $K_{0}$ of characteristic 0 and residue field $k$ of characteristic $p>0$. We will assume that $k$ is algebraically closed. Let $\bar{K}$ be an algebraic closure of $K_{0}$ and $\zeta_{p} \in \boldsymbol{\mu}_{p}(\bar{K})$ a fixed $p$ th root of unity. We write $v_{R_{0}}$ for the valuation of $K_{0}$ corresponding to $R_{0}$. We normalize $v_{R_{0}}$ such that $v_{R_{0}}(p)=1$.

- An integer $m>0$ dividing $p-1$, and a character $\chi: \mathbb{Z} / m \rightarrow \boldsymbol{\mu}_{m}\left(K_{0}\right)$ of order $m$. We write $\bar{\chi}: \mathbb{Z} / m \rightarrow \mathbb{F}_{p}^{\times}$for the reduction of $\chi \bmod v_{R_{0}}$. We define the group $H \cong \mathbb{Z} / p \rtimes \mathbb{Z} / m$ by two generators $\alpha, \beta$ with relations $\alpha^{p}=\beta^{m}=1$ and $\beta^{i} \alpha \beta^{-i}=\alpha^{\bar{\chi}(i)}$.

- An integer $r \geqslant 3$ and an $r$-tuple $\left(a_{1}, \ldots, a_{r}\right)$ of integers such that $\operatorname{gcd}\left(a_{1}, \ldots, a_{r}\right)=1$, $0<a_{i}<m$ and $\sum_{i} a_{i} \equiv 0 \bmod m$.

- An $r$-tuple $\left(x_{1}, \ldots, x_{r}\right)$ of pairwise distinct $K_{0}$-rational points on the projective line $\mathbb{P}^{1}$.

The inverse image of an element $a \in(\mathbb{Z} / m)^{\times}$under the natural map $H \rightarrow \mathbb{Z} / m$ is a conjugacy class of $H$, containing elements of order $m /(m, a)$. We denote this conjugacy class by $C_{a}$.

DeFINITION 1.1. - A metacyclic cover of type $\left(x_{i} ; a_{i}\right)$ is an $H$-cover

$$
f: Y \stackrel{H}{\longrightarrow} \mathbb{P}_{\bar{K}}^{1}
$$

defined over $\bar{K}$, branched in $x_{1}, \ldots, x_{r}$, such that the canonical generator of inertia above $x_{i}$ (with respect to the character $\chi$ ) is an element of $C_{a_{i}}$, for $i=1, \ldots, r$ (see e.g. [18]). The cover $f$ is called of multiplicative type if

$$
\sum_{i=1}^{r} a_{i}=m .
$$

The meaning of the term 'of multiplicative type' will become clear later. For the moment, let us fix a metacyclic cover $f: Y \rightarrow \mathbb{P}^{1}$ of type $\left(x_{i} ; a_{i}\right)$, not necessarily of multiplicative type. Let $Z$ be the quotient of $Y$ by the normal subgroup $\langle\alpha\rangle \triangleleft H$ of order $p$. Thus, $g: Z \rightarrow \mathbb{P}_{\bar{K}}^{1}$ is an $m$ cyclic cover, branched in $x_{1}, \ldots, x_{r}$ such that the image of $\beta^{a_{i}}$ in $H /\langle\alpha\rangle \cong \mathbb{Z} / m$ is the canonical generator of inertia above $x_{i}$. We shall call $g: Z \rightarrow \mathbb{P}^{1}$ the $m$-cyclic cover of type $\left(x_{i} ; a_{i}\right)$, with respect to $\chi$. In concrete terms, $Z$ is the normalization of the plane curve with equation

$$
z^{m}=\prod_{i=1}^{r}\left(x-x_{i}\right)^{a_{i}}
$$

(provided $x_{i} \neq \infty$ ), and the restriction of $\beta \in H$ to $Z$ yields a generator $\tilde{\beta}: Z \stackrel{\sim}{\rightarrow} Z$ of $\operatorname{Gal}\left(Z / \mathbb{P}^{1}\right) \cong \mathbb{Z} / m$ such that $\tilde{\beta}^{*} z=\chi(1) z$.

The $p$-cyclic cover $Y \rightarrow Z$ is étale. Hence it corresponds to a nontrivial class $\theta$ in $H_{\text {et }}^{1}(Z, \mathbb{Z} / p)_{\chi}$ (for any $\mathbb{F}_{p}[\mathbb{Z} / m]$-module $M$, we denote by $M_{\chi}$ the $\chi$-eigenspace). Let $J_{Z}=\operatorname{Pic}^{0}(Z)$ denote the Jacobian of $Z$, and $J_{Z}[p]$ its group of $\bar{K}$-rational points of order $p$. Kummer theory gives us a canonical isomorphism

$$
H_{\text {ét }}^{1}(Z, \mathbb{Z} / p)_{\chi} \cong J_{Z}[p]_{\chi}(-1)=\operatorname{Hom}_{\mathbb{F}_{p}}\left(\boldsymbol{\mu}_{p}(\bar{K}), J_{Z}[p]_{\chi}\right),
$$


see [10], III $\S 4$. Here is how one can define the cover $Y \rightarrow Z$ from a class $\theta \in H_{\text {ét }}^{1}(Z, \mathbb{Z} / p)_{\chi}$. Using our choice of a $p$ th root of unity $\zeta_{p}$, we can identify $J_{Z}[p]_{\chi}(-1)$ with $J_{Z}[p]_{\chi}$. Let $L$ be the line bundle on $Z$ of order $p$ corresponding to the class $\theta$ under the isomorphism (3). Let $D$ be a divisor on $Z$ such that $L \cong \mathcal{O}_{Z}(D)$. Then $p \cdot D$ is the divisor of some rational function $u$ on $Z$. Let $Y \rightarrow Z$ be the cover which is (birationally) given by the equation

$$
y^{p}=u \text {. }
$$

We let the generator $\alpha$ of the $p$-cyclic subgroup of $H$ act on $Y$ such that $\alpha^{*} y=\zeta_{p} y$. By our choice of $D$ we have $\tilde{\beta}^{*} D \equiv \bar{\chi}(1) \cdot D$. Therefore, there exist an integer $\mu$ such that $\mu \equiv \bar{\chi}(1) \bmod p$ and a rational function $v$ on $Z$ such that $\tilde{\beta}^{*} D=\mu \cdot D+(v)$. We can normalize $v$ such that $\tilde{\beta}^{*} u=u^{\mu} v^{p}$. We see that $\tilde{\beta}$ lifts to an automorphism $\beta: Y \stackrel{\sim}{\rightarrow} Y$ of order $m$ such that $\beta^{*} y:=y^{\mu} v$. It is easy to check that this defines an action of $H=\langle\alpha, \beta\rangle$ on $Y$.

\subsection{The field of moduli}

Let $K^{\text {in }}$ be the field of moduli of the $H$-cover $f: Y \rightarrow \mathbb{P}^{1}$, relative to the extension $\bar{K} / K_{0}$ (see e.g. [4]). Since the group $H$ has trivial center, this means that $K^{\text {in }} / K_{0}$ is the smallest field extension such that the $H$-cover $f$ descends to an $H$-cover $f_{K^{\text {in }}}: Y_{K^{\text {in }}} \rightarrow \mathbb{P}_{K^{\text {in }}}^{1}$ over $K^{\text {in }}$. Moreover, the extension $K^{\text {in }} / K_{0}$ is finite, and the model $f_{K^{\text {in }}}$ of $f$ is unique up to $K^{\text {in }}$-linear isomorphism. We let $\Gamma^{\text {in }}:=\operatorname{Gal}\left(\bar{K} / K^{\text {in }}\right)$.

The field of moduli of the $m$-cyclic cover $g: Z \rightarrow \mathbb{P}^{1}$ is just $K_{0}$. Although $g$ has no unique $K_{0}$-model (because $\mathbb{Z} / m$ is abelian), there is a canonical model $g_{K_{0}}: Z_{K_{0}} \rightarrow \mathbb{P}_{K_{0}}^{1}$, given by Eq. (2). (Since $K_{0}$ is strictly henselian, $g_{K_{0}}$ is characterized by the fact that it is unramified at the generic point of $\mathbb{P}_{k}^{1}$. Note, however, that the $m$-cyclic quotient $Y_{K^{\text {in }}} /(\mathbb{Z} / p) \rightarrow \mathbb{P}_{K^{\text {in }}}^{1}$ of $f_{K^{\text {in }}}$ will not be $K^{\text {in }}$-isomorphic to $g_{K_{0}} \otimes K^{\text {in }}$, in general.) The choice of the model $g_{K_{0}}$ determines an action of $\Gamma_{K_{0}}:=\operatorname{Gal}\left(\bar{K} / K_{0}\right)$ on the $\mathbb{F}_{p}[\mathbb{Z} / m]$-module $H_{\text {ét }}^{1}(Y, \mathbb{Z} / p)$, and hence an action on the $\chi$-eigenspace $H_{\text {ét }}^{1}(Z, \mathbb{Z} / p)_{\chi}$. We can describe the subgroup $\Gamma^{\text {in }} \subset \Gamma_{K_{0}}$, and therefore the field extension $K^{\text {in }} / K_{0}$, in terms of this action, as follows.

Proposition 1.2. - Let $\theta \in H^{1}\left(Z, \mathbb{F}_{p}\right)_{\chi}$ be the class corresponding to the étale $p$-cyclic cover $Y \rightarrow Z$. Then

$$
\Gamma^{\text {in }}=\left\{\left.\sigma \in \Gamma_{K_{0}}\right|^{\sigma} \theta=\bar{\chi}(a) \theta, a \in \mathbb{Z} / m\right\} .
$$

Proof. - Let $\sigma \in \Gamma_{K_{0}}$. By definition, $\sigma$ is an element of $\Gamma^{\text {in }}$ if and only if the conjugate cover ${ }^{\sigma} f:{ }^{\sigma} Y \rightarrow \mathbb{P}^{1}$ is isomorphic to $f$. This means that there exists a $\bar{K}$-linear isomorphism $\phi:{ }^{\sigma} Y \stackrel{\sim}{\rightarrow} Y$ which is equivariant with respect to the $H$-action, such that $f \circ \phi={ }^{\sigma} f$. We may (and will) identify ${ }^{\sigma} Z$ with $Z$ (using the model $Z_{K_{0}}$ ). So, if it exists, the isomorphism $\phi$ restricts to a $\bar{K}$-linear automorphism of $Z$ which commutes with the $\mathbb{Z} / m$-action and the map $g: Z \rightarrow \mathbb{P}^{1}$; therefore, $\left.\phi\right|_{Z}=\tilde{\beta}^{a}$, for some $a \in \mathbb{Z} / m$. In other words, $\sigma \in \Gamma^{\text {in }}$ if and only if ${ }^{\sigma} \theta=\left(\tilde{\beta}^{a}\right)^{*} \theta=\bar{\chi}(a) \theta$, for some $a \in \mathbb{Z} / m$. This is what we wanted to prove.

Of course, the choice of the model $g_{K_{0}}$ also determines an action of $\Gamma_{K_{0}}$ on $J_{Z}[p]_{\chi}$ and $J_{Z}[p]_{\chi}(-1)$, such that (3) becomes an isomorphism of $\mathbb{F}_{p}\left[\Gamma_{K_{0}}\right]$-modules. One way to study this action is to regard $J_{Z}[p]_{\chi}$ as the group of $\bar{K}$-rational points of the group scheme $\operatorname{Jac}\left(Z_{K_{0}}\right)[p]_{\bar{\chi}}$ over $K_{0}$. A crucial fact we will use in this paper is the following. If $f$ is of multiplicative type and if the branch points of $f$ are equidistant, then $\operatorname{Jac}\left(Z_{K_{0}}\right)[p]_{\bar{\chi}}$ extends to a constant group scheme over $R_{0}$. Equivalently, $\Gamma_{K_{0}}$ acts trivially on $J_{Z}[p]_{\chi}$. This is a special case of Proposition 1.3 below.

Since we do not want to assume that the branch points of $f$ are equidistant, we need the notion of admissible reduction. This notion was introduced in [7]. Another reference is [15], where 
admissible covers are called 'kummérien'. Let $\left(X_{R_{0}} ; x_{i, R_{0}}\right)$ be the (unique) stably pointed model over $R_{0}$ of the $r$-pointed curve $\left(\mathbb{P}_{K_{0}}^{1} ; x_{i}\right)$, in the sense of [9]. Let $K / K_{0}$ be a finite extension, $R$ the ring of integers of $K$ and $X_{R}:=X_{R_{0}} \otimes R$. Furthermore, let $Z_{R}$ be the normalization of $X_{R}$ in the function field of $Z_{K}:=Z_{K_{0}} \otimes K$ and let $g_{R}: Z_{R} \rightarrow X_{R}$ be the canonical map. We say that $g_{R}$ is an admissible cover if the following holds:

(a) The curve $Z_{R}$ is semistable.

(b) The map $g_{R}: Z_{R} \rightarrow X_{R}$ is finite, and its restriction to the smooth locus of $X_{R}$ is a tame cover, ramified only along the sections $x_{1, R}, \ldots, x_{r, R}$.

(c) Let $x$ be a singular point of the special fiber $\bar{X}:=X_{R} \otimes_{R} k$ and let $z \in \bar{Z}:=Z_{R} \otimes_{R} k$ be a point above $x$. Then $z$ is also a singular point of $\bar{Z}$. Moreover, if $\bar{X}^{\prime}, \bar{X}^{\prime \prime}$ denote the two branches of $\bar{X}$ passing through $x$ and $\bar{Z}^{\prime}, \bar{Z}^{\prime \prime}$ denote their inverse images in $\bar{Z}$, then the canonical generator of inertia of the $m$-cyclic cover $\bar{Z}^{\prime} \rightarrow \bar{X}^{\prime}$ at $z$ is the inverse of the canonical generator of $\bar{Z}^{\prime \prime} \rightarrow \bar{X}^{\prime \prime}$ at $z$.

If $g_{R}$ is an admissible cover, then we say that $g_{K_{0}}$ has admissible reduction over $K / K_{0}$ and call $g_{R}: Z_{R} \rightarrow X_{R}$ the admissible model of $g_{K_{0}}$ over $R$.

Proposition 1.3. - Suppose that $f$ is of multiplicative type, i.e. Eq. (1) holds. Then there exists a finite, at most tamely ramified extension $K / K_{0}$ such that the following holds.

(i) The cover $g_{K_{0}}$ has admissible reduction over $K$.

(ii) Let $\bar{g}: \bar{Z} \rightarrow \bar{X}$ be the special fiber of the admissible model of $g_{K_{0}}$ over $R,\left(\bar{X}_{j}\right)$ the collection of irreducible components of $\bar{X}$ and $\bar{Z}_{j}$ the inverse image of $\bar{X}_{j}$. Then for each $j$, the curve $\bar{Z}_{j}$ is smooth (but not necessarily connected) and the group scheme $\operatorname{Jac}\left(\bar{Z}_{j}\right)[p]_{\bar{\chi}}$ is étale.

(iii) Specialization yields an isomorphism

$$
J_{Z}[p]_{\bar{\chi}}(\bar{K}) \stackrel{\sim}{\longrightarrow} \bigoplus_{j} \operatorname{Jac}\left(\bar{Z}_{j}\right)[p]_{\bar{\chi}}(k)
$$

of $\mathbb{F}_{p}[\mathbb{Z} / m]$-modules.

If, moreover, $x_{i} \not \equiv x_{j} \bmod v_{R_{0}}$ for $i \neq j$ then $\bar{Z}$ is smooth and we can take $K:=K_{0}$. Furthermore, $\operatorname{Jac}\left(Z_{K_{0}}\right)[p]_{\bar{\chi}}$ is isomorphic, as group scheme over $K_{0}$, to the constant group scheme $(\mathbb{Z} / p)^{r-2}$.

To prove this proposition, we need the following lemma. Its proof follows a suggestion of Prof. Raynaud.

Lemma 1.4. - Let $k, p, m$ and $\bar{\chi}$ be as at the beginning of Section 1.1. Let $\bar{x}_{1}, \ldots, \bar{x}_{r}$ be pairwise distinct elements of $k$. Let $a_{1}, \ldots, a_{r}$ be integers such that $0<a_{i}<m$ and $\sum_{i} a_{i}=m$ (we do not assume that $\left.\operatorname{gcd}\left(a_{i}\right)=1\right)$. Let $\bar{g}: \bar{Z} \rightarrow \mathbb{P}_{k}^{1}$ be the $m$-cyclic cover of type $\left(\bar{x}_{i} ; a_{i}\right)$, with respect to $\bar{\chi}$ (this makes sense even if $\operatorname{gcd}\left(a_{i}\right) \neq 1$ in which case $\bar{Z}$ is not connected). Then

(i) $H^{1}\left(\bar{Z}, \mathcal{O}_{\bar{Z}}\right)_{\bar{\chi}}=0$.

(ii) $\operatorname{dim}_{k} H^{0}\left(\bar{Z}, \Omega_{\bar{Z} / k}\right)_{\bar{\chi}}=r-2$.

(iii) The group scheme $\operatorname{Jac}(\bar{Z})[p]_{\bar{\chi}}$ is étale, isomorphic to $(\mathbb{Z} / p)^{r-2}$.

(iv) The Cartier operator is a bijection on $H^{0}\left(\bar{Z}, \Omega_{\bar{Z} / k}\right)_{\bar{\chi}}$.

Proof. - We may identify $\bar{Z}$ with the nonsingular model of the plane curve $z^{m}=\prod_{i}\left(x-\bar{x}_{i}\right)^{a_{i}}$ over $k$. The action of $\mathbb{Z} / m$ on $\bar{Z}$ induces an action on the sheaf $\bar{g}_{*} \mathcal{O}_{\bar{Z}}$; let

$$
\bar{g}_{*} \mathcal{O}_{\bar{Z}}=\bigoplus_{\bar{\psi}} \mathcal{L}_{\bar{\psi}}
$$


be the decomposition into isotypical subsheaves, where $\bar{\psi}$ runs through all characters $\mathbb{Z} / m \rightarrow k^{\times}$. It is clear that $\mathcal{L}_{\bar{\psi}}$ is a line bundle, for all $\bar{\psi}$. By assumption, the rational function $z$ is a rational section of $\mathcal{L}_{\bar{\chi}}$ which defines a trivialization $\left.\mathcal{L}_{\bar{\psi}}\right|_{\mathbb{A}_{k}^{1}} \cong \mathcal{O}_{\mathbb{A}_{k}^{1}}$ and has a pole of order $\left(\sum_{i} a_{i}\right) / m=1$ at $\infty$. We conclude that

$$
\operatorname{dim}_{k} H^{1}\left(\bar{Z}, \mathcal{O}_{\bar{Z}}\right)_{\bar{\chi}}=\operatorname{dim}_{k} H^{1}\left(\mathbb{P}_{k}^{1}, \mathcal{L}_{\bar{\chi}}\right)=\operatorname{deg} \mathcal{L}_{\bar{\chi}}-1=0 .
$$

This proves (i). A similar calculation (using the function $z^{m-1}$ instead of $z$ ) shows that the $k$-dimension of $H^{1}\left(\bar{Z}, \mathcal{O}_{\bar{Z}}\right)_{\bar{\chi}^{-1}}$ is $r-2$. But $H^{1}\left(\bar{Z}, \mathcal{O}_{\bar{Z}}\right)_{\bar{\chi}^{-1}}$ is dual to $H^{0}\left(\bar{Z}, \Omega_{\bar{Z} / k}\right)_{\bar{\chi}}$, so (ii) follows. Let $\bar{J}$ be the Jacobian of the curve $\bar{Z}$. We have a canonical $k$-linear isomorphism

$$
\operatorname{Lie}(\bar{J}[p])=\operatorname{Lie}(\bar{J}) \cong H^{1}\left(\bar{Z}, \mathcal{O}_{\bar{Z}}\right),
$$

see [11], p. 147. This isomorphism is compatible with the natural $\mathbb{Z} / m$-action on both sides and therefore induces an isomorphism on the $\bar{\chi}$-eigenspaces. From (i) we conclude

$$
\operatorname{Lie}(\bar{J}[p])_{\bar{\chi}} \cong H^{1}\left(\bar{Z}, \mathcal{O}_{\bar{Z}}\right)_{\bar{\chi}}=0 .
$$

This shows that $\bar{J}[p]_{\bar{\chi}}$ is an étale group scheme. The rank of $\bar{J}[p]_{\bar{\chi}}$ is equal to

$$
\operatorname{dim}_{k} H_{\mathrm{dR}}^{1}(\bar{Z})_{\bar{\chi}}=\operatorname{dim}_{k} H^{1}\left(\bar{Z}, \mathcal{O}_{\bar{Z}}\right)_{\bar{\chi}}+\operatorname{dim}_{k} H^{0}\left(\bar{Z}, \Omega_{\bar{Z} / k}\right)_{\bar{\chi}}=r-2,
$$

by (i) and (ii). This proves (iii). The canonical polarization on $\bar{J}$ identifies $\bar{J}[p]_{\bar{\chi}}$ with the Cartier dual of $\bar{J}[p]_{\bar{\chi}^{-1}}$. Hence (iii) implies $\bar{J}[p]_{\bar{\chi}^{-1}} \cong\left(\boldsymbol{\mu}_{p}\right)^{r-2}$. Under the isomorphism

$$
H^{1}\left(\bar{Z}, \mathcal{O}_{\bar{Z}}\right)_{\bar{\chi}^{-1}} \cong \operatorname{Lie}(\bar{J}[p])_{\bar{\chi}^{-1}} \cong \operatorname{Lie}\left(\boldsymbol{\mu}_{p}\right)^{r-2},
$$

the action of Frobenius on cohomology corresponds to the $p$ th power map on the Lie algebra, see [11], Theorem 15.3. It is elementary to see that the $p$ th power map on $\operatorname{Lie}\left(\boldsymbol{\mu}_{p}\right)$ is a bijection. Therefore, the Frobenius is a bijection on $H^{1}\left(\bar{Z}, \mathcal{O}_{\bar{Z}}\right)_{\bar{\chi}^{-1}}$. But the transpose of Frobenius with respect to Serre duality is the Cartier operator (see [16]), so (iv) follows. This finishes the proof of Lemma 1.4.

Proof of Proposition 1.3. - We will proceed in two steps. First, let us assume that $x_{i} \not \equiv x_{j} \bmod v_{R_{0}}$ for $i \neq j$. Then the $m$-cyclic cover $g_{K_{0}}: Z_{K_{0}} \rightarrow \mathbb{P}_{K_{0}}^{1}$ extends to a tame $\mathbb{Z} / m$-cover $g_{R_{0}}: Z_{R_{0}} \rightarrow \mathbb{P}_{R_{0}}^{1}$. In particular, $Z_{R_{0}}$ is smooth over $R_{0}$. Let $J_{R_{0}}$ be the Jacobian of $Z_{R_{0}}$ and $\bar{J}$ its special fiber. Then $\bar{J}$ is the Jacobian of the special fiber $\bar{Z}$ of $Z_{R_{0}}$. By Lemma 1.4(iii), $\bar{J}[p]_{\bar{\chi}}$ is étale, isomorphic to $(\mathbb{Z} / p)^{r-2}$. Since $K_{0}$ is strictly henselian, this implies that $\operatorname{Jac}\left(Z_{K_{0}}\right)[p]_{\bar{\chi}}$ is a constant group scheme over $K_{0}$, isomorphic to $(\mathbb{Z} / p)^{r-2}$. This proves the proposition in the case $x_{i} \not \equiv x_{j} \bmod v_{R_{0}}$.

We now consider the general case. Since $m$ is prime to $p$, it follows from the results of [15] that the cover $g_{K_{0}}$ has admissible reduction over $K$, where $K / K_{0}$ is any sufficiently large, tamely ramified extension. This proves Part (i) of Proposition 1.3. Let $g_{R}: Z_{R} \rightarrow X_{R}$ be the admissible model of $g_{K_{0}}$ over $R$ and $\bar{g}: \bar{Z} \rightarrow \bar{X}$ the special fiber of $g_{R}$. By the definition of admissibility, $\bar{Z}$ and $\bar{X}$ are semistable curves and the map $\bar{g}$ is finite and étale, except above the singular points of $\bar{X}$ and the specializations of the branch points $x_{i}$. Let $\left(\bar{X}_{j}\right)$ be the collection of irreducible components of $\bar{X}$ and $\bar{Z}_{j}$ the inverse image of $\bar{X}_{j}$ in $\bar{Z}$. Also by the definition of admissibility, $\bar{Z}_{j} \rightarrow \bar{X}_{j}$ is a (possibly disconnected) $m$-cyclic cover of type $\left(\bar{x}_{j, l} ; a_{j, l}\right)$. Here $\bar{x}_{j, 1}, \ldots, \bar{x}_{j, r_{j}}$ are the points on $\bar{X}_{j}$ which are either singular on $\bar{X}$ or the specialization of a branch point $x_{i}$. We 
may assume that $0 \leqslant a_{j, l} \leqslant m$. If $\bar{x}_{j, l}$ is the specialization of $x_{i}$ then $a_{j, l}=a_{i}$. Moreover, if two components $\bar{X}_{j}$ and $\bar{X}_{j^{\prime}}$ intersect in the point $x_{j, l}=x_{j^{\prime}, l^{\prime}}$ then $a_{j^{\prime}, l^{\prime}}=m-a_{j, l}$. It is now an easy exercise to show that the equality $\sum_{i} a_{i}=m$ and the strict inequality $0<a_{i}<m$ imply the equality

$$
\sum_{l=1}^{r_{j}} a_{j, l}=m
$$

for all $j$ and the strict inequality $0<a_{j, l}<m$ for all $j, l$. Moreover, we have

$$
\sum_{j}\left(r_{j}-2\right)=r-2
$$

(To prove (5) and (6) we use the fact that the graph of components of $\bar{X}$ is a tree; compare with Lemma 2.3 and Proposition 2.4.) From (5) and Lemma 1.4 we conclude that $\operatorname{Jac}\left(\bar{Z}_{j}\right)[p]_{\bar{\chi}}$ is étale of rank $p^{r_{j}-2}$. This shows (ii).

Let $K^{\prime} / K$ be a finite extension, $Z_{K^{\prime}}:=Z_{K_{0}} \otimes_{K_{0}} K^{\prime}$ and $J_{K^{\prime}}$ the Jacobian of $Z_{K^{\prime}}$. We may choose $K^{\prime}$ such that all points of the group scheme $J_{K^{\prime}}[p]_{\bar{\chi}}$ are $K^{\prime}$-rational. As in the proof of Lemma 1.4 one shows that

$$
J_{K^{\prime}}[p]_{\bar{\chi}} \cong(\mathbb{Z} / p)^{r-2} .
$$

Let $J_{R^{\prime}}$ be the Néron model of $J_{Z^{\prime}}$. It follows from our assumption and the universal property of the Néron model that $\mathcal{G}:=J_{R^{\prime}}[p]_{\bar{\chi}}$ is a finite and flat group scheme over $R^{\prime}$, with generic fiber $J_{K^{\prime}}[p]_{\bar{\chi}}$. Let $\bar{J}:=J_{R^{\prime}} \otimes k$ be the special fiber of $J_{R^{\prime}}$ and $\bar{J}^{0}$ its connected component containing 0 . Since $Z_{K^{\prime}}$ has semi-stable reduction, $\bar{J}^{0}$ is a semi-abelian variety and sits inside a short exact sequence

$$
0 \rightarrow T \longrightarrow \bar{J}^{0} \longrightarrow \bigoplus_{j} \mathrm{Jac}\left(\bar{Z}_{j}\right) \rightarrow 0
$$

Here $J_{\bar{Z}_{j}}$ is the Jacobian of $\bar{Z}_{j}$ and $T$ is an algebraic torus over $k$. See [1]. The group $\mathbb{Z} / m$ acts on all terms of (8). In particular, we can define the $\bar{\chi}$-isotypical subtorus $T_{\bar{\chi}} \subset T$. Furthermore, $\mathbb{Z} / m$ acts on the finite abelian group $\bar{J} / \bar{J}^{0}$, and we let $\left(\bar{J} / \bar{J}^{0}\right)[p]_{\bar{\chi}}$ be the $\bar{\chi}$-isotypical part of the group of elements of order $p$ in $\bar{J} / \bar{J}^{0}$. By (7) and (8) we have

$$
\sum_{j}\left(r_{j}-2\right)+\operatorname{dim} T_{\bar{\chi}}+\frac{\log \left|\left(\bar{J} / \bar{J}^{0}\right)[p]_{\bar{\chi}}\right|}{\log p}=r-2 .
$$

With (6) we conclude that $\left(\bar{J} / \bar{J}^{0}\right)[p]_{\bar{\chi}}=0$ and that $T_{\bar{\chi}}=1$. In other words, we have

$$
\mathcal{G} \otimes k \cong \bigoplus_{j} \operatorname{Jac}\left(\bar{Z}_{j}\right)[p]_{\bar{\chi}}
$$

This proves (iii).

COROLLARY 1.5. - If $f$ is of multiplicative type, then $f$ has bad reduction, and $K^{\mathrm{in}} / K_{0}$ is at most tamely ramified. If, moreover, $x_{i} \not \equiv x_{j} \bmod v_{R_{0}}$ for all $i \neq j$ then

$$
K^{\text {in }}=K_{0}\left(\zeta_{p}^{(m)}\right):=K_{0}\left(\zeta_{p}\right)^{\mathbb{Z} / m}
$$

(where $a \in \mathbb{Z} / m$ acts on $K_{0}\left(\zeta_{p}\right)$ as $\zeta_{p} \mapsto \zeta_{p}^{\bar{\chi}(a)}$ ).

$4^{\mathrm{e}}$ SÉRIE - TOME $36-2003-\mathrm{N}^{\circ} 1$ 
Proof. - The statement about $K^{\text {in }}$ follows directly from Proposition 1.2 and Proposition 1.3. It follows from [12] and Proposition 1.3 that the $p$-cyclic cover $Y \rightarrow Z$ reduces to a $\boldsymbol{\mu}_{p}$-torsor over the generic point of $\bar{Z}_{j}$. In particular, if $\bar{Y}$ is the special fiber of a semistable model of $Y$ which admits a finite map $\bar{Y} \rightarrow \bar{Z}$, then this map will be inseparable. This means that $f$ has bad reduction.

\subsection{Special covers}

For the rest of this section, we will assume that $f: Y \rightarrow \mathbb{P}^{1}$ is a metacyclic cover of multiplicative type $\left(x_{i} ; a_{i}\right)$. We do not assume that $x_{i} \not \equiv x_{j} \bmod v_{R_{0}}$. By Corollary $1.5, f$ has bad reduction. Let $K^{\text {in }} / K_{0}$ be the field of moduli of $f$ and $f_{K^{\text {in }}}: Y_{K^{\text {in }}} \rightarrow \mathbb{P}_{K^{\text {in }}}^{1}$ the model of $f$ over $K^{\text {in }}$. Furthermore, let $K / K^{\text {in }}$ be a sufficiently large finite extension over which $f_{K^{\text {in }}}$ has stable reduction, see [20]. By this we mean the following, compare with [13] and [3]. First, the ramification points $y_{1}, \ldots, y_{s} \in Y$ of the $H$-cover $f$ are $K$-rational points on $Y_{K^{\text {in }}}$. Second, after the base change $Y_{K}:=Y_{K^{\text {in }}} \otimes_{K^{\text {in }}} K$, the pointed curve $\left(Y_{K} ; y_{i}\right)$ extends to a stably pointed curve $\left(Y_{R} ; y_{R, i}\right)$ over the ring of integers $R$ of $K$. The quotient map $f_{R}: Y_{R} \rightarrow X_{R}:=Y_{R} / H$ is called the stable model of $f$ over $R$. Its special fiber $\bar{f}: \bar{Y} \rightarrow \bar{X}$ is called the stable reduction of the $H$-cover $f$. Note that $\bar{f}$ is a finite map between pointed semistable $k$-curves. Here the distinguished points on $\bar{Y}$ are the specializations $\bar{y}_{1}, \ldots, \bar{y}_{s}$ of the ramification points, and the distinguished points on $\bar{X}$ are the specializations $\bar{x}_{1}, \ldots, \bar{x}_{r}$ of the branch points of $f$. However, $\left(\bar{X} ; \bar{x}_{i}\right)$ is not a stably pointed curve, in general.

We call a component $\bar{X}^{\prime}$ of $\bar{X}$ separable if the restriction of $\bar{f}$ to one (and therefore to all) components of $\bar{Y}$ above $\bar{X}^{\prime}$ is a separable morphism. Note that $\bar{X}$ has at least one component that is not separable, by the definition of bad reduction. Let us denote by $\bar{X}_{i}$ the component of $\bar{X}$ containing $\bar{x}_{i}$.

For convenience, we make the following assumption, which will be part of the definition of a special cover (Definition 1.9 below).

Assumption 1.6. - (i) A component $\bar{X}^{\prime}$ of $\bar{X}$ is separable if and only if it is a tail, i.e. if it is connected to the rest of $\bar{X}$ in a single point.

(ii) The components $\bar{X}_{1}, \ldots, \bar{X}_{r}$ are pairwise distinct, and they are precisely the tails of $\bar{X}$.

Remark 1.7. - It is easy to see that the components $\bar{X}_{i}$ are separable. If the branch points of $f$ do not coalesce, i.e. $x_{i} \not \equiv x_{j} \bmod v_{R_{0}}$ for $i \neq j$, then part (i) of Assumption 1.6 holds automatically, by [13], Lemme 3.1.2. In this case, Assumption 1.6(ii) can be phrased (in the terminology of [13]) as: 'there are no new tails'. One should keep in mind that this is a rather strong condition.

Fix $i \in\{1, \ldots, r\}$. The restriction of $\bar{f}$ to the tail $\bar{X}_{i}$ is a (possibly disconnected) $H$-Galois cover $\bar{f}_{i}: \bar{Y}_{i} \rightarrow \bar{X}_{i}$. The cover $\bar{f}_{i}$ is ramified at exactly two points, namely at $\bar{x}_{i}$ and at the unique intersection point of $\bar{X}_{i}$ with the rest of $\bar{X}$ (let us call this point $\tau_{i}$ ). The ramification above $\bar{x}_{i}$ is tame and, since $\bar{x}_{i}$ is the specialization of the branch point $x_{i}$ of $f$, the canonical generator of inertia above $\bar{x}_{i}$ is in the conjugacy class $C_{a_{i}}$. On the other hand, the ramification at $\tau_{i}$ is wild, of order $p m_{i}$, where $m_{i}:=m /\left(a_{i}, m\right)$. The cover $\bar{f}_{i}: \bar{Y}_{i} \rightarrow \bar{X}_{i} \cong \mathbb{P}_{k}^{1}$ is of 'Katz-Gabber-type'; see e.g. [5], §2, where such covers are called ' $m_{i}$-special'. We set $\sigma_{i}:=h_{i} / m_{i}$, where $h_{i}$ is the conductor of the $p$-part of inertia. Recall that $\sigma_{i}$ is the jump in the inertia group filtration of the cover $\bar{f}_{i}: \bar{Y}_{i} \rightarrow \bar{X}_{i}$, with respect to the upper numbering (see [17]).

PROPOSITION 1.8. - There exist integers $\nu_{i} \geqslant 0, i=1, \ldots, r$, such that

(i) $\sigma_{i}=\nu_{i}+a_{i} / m$, and

(ii) $\sum_{i=1}^{r} \nu_{i}=r-3$. 
Proof. - Let us fix an index $i$ and set $d_{i}:=\left(m, a_{i}\right)$. The cover $\bar{Z}_{i}:=\bar{Y}_{i} /\langle\alpha\rangle \rightarrow \bar{X}_{i}$ is a (possibly disconnected) $\mathbb{Z} / m$-cover of type $\left(\bar{x}_{i}, \tau_{i} ; a_{i}, m-a_{i}\right)$ with respect to $\bar{\chi}$ (see Section 1.1). Let $\xi_{i} \in \bar{Z}_{i}$ be any point above $\tau_{i}$ and let $w$ be a local parameter at $\xi_{i}$. In a neighborhood of $\xi_{i}$, the $p$-cyclic cover $\bar{Y}_{i} \rightarrow \bar{Z}_{i}$ admits a representation as an Artin-Schreier cover

$$
y^{p}-y=w^{-h_{i}},
$$

such that $\alpha^{*} y=y+1$. The congruence

$$
\left(\beta^{m-a_{i}}\right)^{*} w \equiv \bar{\chi}\left(d_{i}\right) \cdot w \quad \bmod w^{2}
$$

implies the congruence

$$
\left(\beta^{m-a_{i}}\right)^{*} y \equiv \bar{\chi}\left(-h_{i} d_{i}\right) \cdot y+\eta \bmod w^{2}
$$

for some $\eta \in \mathbb{F}_{p}$. The rule $\beta \alpha \beta^{-1}=\alpha^{\bar{\chi}(1)}$ and a short calculation show that

$$
m-a_{i} \equiv-h_{i} d_{i} \quad \bmod m .
$$

This proves (i), i.e. $\sigma_{i}=\nu_{i}+a_{i} / m$, for integers $\nu_{i} \geqslant 0$. On the other hand, we have Raynaud's vanishing cycle formula, [13], §3.4.2 (5). In our situation, it becomes

$$
\sum_{i=1}^{r}\left(1-\sigma_{i}\right)=2 .
$$

Now (ii) follows from (i) and (9).

Here comes the central definition of this paper.

DEFINITION 1.9. - Let $f: Y \rightarrow \mathbb{P}^{1}$ be a metacyclic cover of multiplicative type $\left(x_{i} ; a_{i}\right)$, with (bad) reduction $\bar{f}: \bar{Y} \rightarrow \bar{X}$. Suppose Assumption 1.6 holds, and let $\sigma_{i}=\nu_{i}+a_{i} / m$ be the invariant attached to the wild ramification above the tail $\bar{X}_{i}$, as above. We say that $f$ is special if there are exactly three indices $i_{1}, i_{2}, i_{3}$ with $\nu_{i_{j}}=0$ (therefore, $\nu_{i}=1$ for $i \notin\left\{i_{1}, i_{2}, i_{3}\right\}$, by Proposition 1.8).

Thus, after reordering the branch points $x_{i}$, we may assume that $\nu_{1}, \nu_{2}, \nu_{3}=0$ and $\nu_{i}=1$ for $i=4, \ldots, r$.

\subsection{The auxiliary cover of a three point cover is special}

In this subsection we discuss briefly the example that motivated our study of special covers. For more details, and for arithmetic applications of the results obtained in the present paper, see [20].

Let $G$ be a finite, center free group such that $p$ strictly divides the order of $G$. Let $h: W \rightarrow \mathbb{P}^{1}$ be a $G$-cover over $\bar{K}$, ramified in 0,1 and $\infty$, with ramification indices of order prime-to- $p$. Assume that the cover $h$ has bad reduction. Let $h_{R}: W_{R} \rightarrow X_{R}$ be its stable model and $\bar{h}: \bar{W} \rightarrow \bar{X}$ the special fiber of $h_{R}$. By [13], §3.1, the map $\bar{h}$ is separable exactly over the tails of $\bar{X}$. Let $\bar{X}_{1}, \bar{X}_{2}, \bar{X}_{3}$ be the primitive tails, i.e. those which contain the specialization of the branch points $0,1, \infty$, respectively. Let $\bar{X}_{4}, \ldots, \bar{X}_{r}$ be the new tails. As above, we associate to each tail $\bar{X}_{i}$ the invariant $\sigma_{i}=h_{i} / m_{i}$. By [13], Proposition 3.3.5, we have

$$
\sigma_{i}>1, \quad \text { for } i=4, \ldots, r .
$$


For $i=4, \ldots, r$, we choose a smooth point $\bar{x}_{i}$ on $\bar{X}_{i}$, and lift it to a point $x_{i}$ of $\mathbb{P}_{K}^{1}$. Set $x_{1}:=0$, $x_{2}:=1, x_{3}:=\infty$.

Now let $f: Y \rightarrow \mathbb{P}^{1}$ be the auxiliary cover associated to $h$, see [13]. The cover $f$ is metacyclic of type $\left(x_{i} ; a_{i}\right)$, with $m$ and $\left(a_{1}, \ldots, a_{r}\right)$ as at the beginning of Section 1.1. Furthermore, $f$ has bad reduction, and the special fiber of its stable model is of the form $\bar{f}: \bar{Y} \rightarrow \bar{X}$ (where $\bar{X}$ is the target curve of $\bar{h}$ ). Finally, the tails $\bar{X}_{i}$ of $\bar{X}$ are exactly the separable components (for the map $\bar{f}$ ), and the induced maps $\bar{f}_{i}: \bar{Y}_{i} \rightarrow \bar{X}_{i}$ over the tails are (possibly disconnected) $H$-Galois covers of Katz-Gabber type, tamely ramified at $\bar{x}_{i}$ and wildly ramified at $\tau_{i}$, with invariant $\sigma_{i}$.

We claim that $f$ is special. Indeed, Assumptions 1.6(i) and (ii) hold by construction (except maybe for $f$ being of multiplicative type, i.e. $\sum_{i} a_{i}=m$ ). As in the proof of Proposition 1.8, we show that $\sigma_{i}=\nu_{i}+a_{i} / m$, with integers $\nu_{i} \geqslant 0$ such that

$$
\sum_{i} \nu_{i}=r-2-\frac{\sum_{i} a_{i}}{m} .
$$

But $\nu_{i}>0$ for $i=4, \ldots, r$, by (10), so the left hand side of (11) is $\geqslant r-3$. We conclude that $\sum_{i} a_{i}=m, \nu_{i}=0$ for $i=1,2,3$ and $\nu_{i}=1$ for $i=4, \ldots, r$. In other words, $f$ is special.

It is very likely that every special $H$-cover $f: Y \rightarrow \mathbb{P}^{1}$ arises as the auxiliary cover of a $G$-cover $h: W \rightarrow \mathbb{P}^{1}$ with three branch points, for some group $G$. At the moment, we can prove this claim only modulo the following hypothesis.

Hypothesis 1.10. - Let $p$ be a prime and $m, h>1$ integers such that $m \mid p-1,(h, p)=1$ and $1<\sigma:=h / m<2$. Then there exists a finite group $G$ and an étale $G$-cover $f: W \rightarrow \mathbb{A}_{k}^{1}$ of the affine line, defined over $k=\overline{\mathbb{F}}_{p}$, such that the inertia group at infinity is isomorphic to $\mathbb{Z} / p \rtimes \mathbb{Z} / m$ and the jump in the inertia group filtration is equal to $\sigma=h / m$.

The hypothesis implies the claim, essentially because we can 'reverse' the auxiliary cover construction, using formal patching. This fact establishes a strong link between the existence of étale Galois covers of the affine line in characteristic $p$ with prescribed ramification at infinity (i.e. certain generalized forms of Abhyankar's Conjecture) and the reduction of Galois covers from characteristic 0 to characteristic $p$. For results in this direction, see [2]. These results show that we cannot expect Hypothesis 1.10 to hold for a given group $G$ satisfying the obvious restrictions (quasi- $p$, contains $H$ ).

\section{The structure of special covers}

The main goal of this section is to prove the following result, which essentially states that (the reduction of) a special cover is as simple as we can expect it to be.

THEOREM 2.1. - Let $f: Y \rightarrow \mathbb{P}^{1}$ be a special metacyclic cover of type $\left(x_{i} ; a_{i}\right)$, with reduction $\bar{f}: \bar{Y} \rightarrow \bar{X}$. Then the curve $\bar{X}$ is the union of $r+1$ components $\bar{X}_{0}, \bar{X}_{1}, \ldots, \bar{X}_{r}$, such that $\bar{X}_{0}$ meets $\bar{X}_{i}$ in exactly one point, and $\bar{X}_{i}$ is the component to which $x_{i}$ specializes. In particular, $x_{i} \not \equiv x_{j} \bmod v_{R_{0}}$ (as points on $\mathbb{P}^{1}$ ) for $i \neq j$.

This was called Theorem A in the introduction. Here is a brief outline of the proof. Using results of [14] (which essentially reformulate earlier results of $[12,6]$ and [8] in a form suitable for us), we analyze the stable reduction of the $p$-cyclic part $Y \rightarrow Z$ of a given special cover $f: Y \rightarrow \mathbb{P}^{1}$. We find that, in case Theorem 2.1 did not hold, one of the components of the special fiber $\bar{Y}$ of $Y$ would be an $\boldsymbol{\alpha}_{p}$-torsor. Moreover, this $\boldsymbol{\alpha}_{p}$-torsor would have to satisfy certain strict numerical conditions, coming from specialty of $f$ and compatibility with the $\mathbb{Z} / m$-action. Then a direct calculation shows that such an $\boldsymbol{\alpha}_{p}$-torsor cannot exist, and Theorem 2.1 follows. 
In Sections 2.1 and 2.2 we analyze the stable reduction of $f$ and translate its properties into a combinatorial language similar to the notion of a Hurwitz tree developed in [8]. Here we work under slightly more general assumptions than is actually necessary for the proof of Theorem 2.1. This will be useful in Section 3.

In Section 2.3 we prove the key lemma which implies Theorem 2.1. In Section 2.4 we state a strengthening of Theorem 2.1, which concerns the monodromy action on the special fiber of a special cover.

\subsection{Admissible reduction of $Z \rightarrow \mathbb{P}^{1}$}

Let $f: Y \rightarrow \mathbb{P}^{1}$ be a metacyclic cover of multiplicative type $\left(x_{i} ; a_{i}\right)$. Concerning the reduction of $f$, we use the notation introduced in Section 1.3. In particular, $f_{R}: Y_{R} \rightarrow X_{R}$ is the stable model of $f$, defined over a sufficiently large finite extension $R / R_{0}$, with special fiber $\bar{f}: \bar{Y} \rightarrow \bar{X}$. The branch point $x_{i}$ specializes to a point $\bar{x}_{i} \in \bar{X}$, and $\bar{X}_{i}$ denotes the component of $\bar{X}$ containing $\bar{x}_{i}$. We make the following assumption, which is slightly weaker than Assumption 1.6. In particular, it holds if $f$ is special.

Assumption 2.2. - (i) The map $\bar{f}: \bar{Y} \rightarrow \bar{X}$ is separable exactly over the tails of $\bar{X}$.

(ii) The components $\bar{X}_{1}, \ldots, \bar{X}_{r}$ are pairwise distinct tails.

We define a tree $T$ associated to the semistable curve $\bar{X}$, as follows. The set of vertices $V$ of $T$ is defined as the set of (irreducible) components of $\bar{X}$. For $v \in V$, we refer to the corresponding component of $\bar{X}$ as $\bar{X}_{v}$. The set of edges $E$ of $T$ is, by definition, the set of triples $e=\left(\bar{x}_{e}, v, v^{\prime}\right)$, where $\bar{x}_{e} \in \bar{X}$ is a singular point and $v, v^{\prime} \in V$ are vertices such that $\bar{x}_{e} \in \bar{X}_{v} \cap \bar{X}_{v^{\prime}}$. The assignments $s(e):=v$ and $t(e):=v^{\prime}$ define the source map $s: E \rightarrow V$ and the target map $t: E \rightarrow V$. The edge $\bar{e}:=\left(\bar{x}_{e}, v^{\prime}, v\right) \in E$ is called the opposite edge of $e$.

We define $B \subset V$ as the set of leaves of $T$, i.e. the vertices corresponding to the tails of $\bar{X}$. In other words, for each $v \in B$ there is a unique edge $e_{v}$ such that $t\left(e_{v}\right)=v$. We call the elements of $V^{\prime}:=V-B$ the interior vertices. By Assumption 2.2(ii), we may identify the set $I:=\{1, \ldots, r\}$ with the corresponding subset of $B$. Note that $B=I$ if $f$ is special.

Let us state the following easy lemma without proof.

LEMMA 2.3. - There exists a unique map $E \rightarrow\{0, \ldots, m\}, e \mapsto a_{e}$, such that

(i) $a_{e}=a_{i}$, if $i:=t(e) \in I$, and $a_{e}=0$ if $v:=t(e) \in B-I$,

(ii) for all interior vertices $v$, we have $\sum_{s(e)=v} a_{e}=m$, and

(iii) $a_{\bar{e}}+a_{e}=m$, for all edges $e \in E$.

If $f$ is special, then $0<a_{e}<m$ for all $e \in E$.

Let $Z_{R}:=Y_{R} /(\mathbb{Z} / p)$ be the quotient of $Y_{R}$ by the $p$-cyclic normal subgroup of $H$. It is well known that $Z_{R}$ is again a semistable curve over $R$, with generic fiber $Z_{K}$. Also, the action of $\mathbb{Z} / m$ on $Z$ extends to $Z_{R}$, and the (tamely ramified) $m$-cyclic cover $g_{K}: Z_{K} \rightarrow \mathbb{P}_{K}$ extends to a finite morphism $g_{R}: Z_{R} \rightarrow X_{R}$ of semistable $R$-curves. Since, by Assumption 2.2(ii), the branch points $x_{i}$ of $g_{K}$ specialize to pairwise distinct points of $\bar{X}$, it follows that the cover $g_{R}: Z_{R} \rightarrow X_{R}$ is an $m$-cyclic admissible cover over $R$, i.e. satisfies Conditions (a), (b) and (c) of Section 1.2. (However, $g_{R}$ is not exactly the admissible model of $g_{K_{0}}$ as defined in Section 1.2, because $X_{R}$ is not the stably pointed model of the pointed curve $\left(\mathbb{P}_{K}^{1}, x_{i}\right)$.) For $v \in V$, we set $\bar{Z}_{v}:=\bar{g}^{-1}\left(\bar{X}_{v}\right)$.

Proposition 2.4. - For each vertex $v \in V$, the restriction $\bar{g}_{v}: \bar{Z}_{v} \rightarrow \bar{X}_{v}$ of $\bar{g}$ to $\bar{Z}_{v}$ is $a$ (possibly disconnected) tamely ramified $m$-cyclic cover. If $v$ is an interior vertex, then $\bar{g}_{v}$ is branched at most at the points $\bar{x}_{e} \in \bar{X}_{v}$, with $s(e)=v$. The canonical generator of inertia above $\bar{x}_{e}$ (with respect to $\bar{\chi}$ ) is $a_{e}$, where $a_{e}$ is as in Lemma 2.3. In other words, $\bar{g}_{v}: \bar{Z}_{v} \rightarrow \bar{X}_{v} \cong \mathbb{P}_{k}^{1}$ is the $m$-cyclic cover of type $\left(\bar{x}_{e} ; a_{e}\right)$. 
For $i \in I$, the cover $\bar{Z}_{i} \rightarrow \bar{X}_{i}$ is ramified at the two points $\bar{x}_{i}$ and $\bar{x}_{e}$ (where $e$ is the unique edge with $s(e)=i)$. The canonical generator of inertia above $\bar{x}_{i}\left(\right.$ resp. $\left.\bar{x}_{e}\right)$ is $a_{i}\left(\right.$ resp. $\left.a_{e}=m-a_{i}\right)$. Finally, for $v \in B-I$, the cover $\bar{Z}_{v} \rightarrow \bar{X}_{v}$ is totally disconnected, i.e. identifies $\bar{Z}_{v}$ with $m$ disjoint copies of $\bar{X}_{v}$.

Proof. - By the definition of admissibility, the covers $\bar{g}_{v}: \bar{Z}_{v} \rightarrow \bar{X}_{v}$ are as in the statement of the proposition, for certain integers $a_{e}$. Moreover, the integers $a_{e}$ verify condition (i), (ii) and (iii) of Lemma 2.3, at least modulo $m$. Now the statement of Lemma 2.3 says that we may choose the $a_{e}$ 's such that they verify condition (i), (ii) and (iii) exactly.

\subsection{The reduction of the $p$-cyclic cover $Y \rightarrow Z$}

We continue with the notation and assumptions of the previous subsection. Recall from Section 1.1 that the étale $p$-cyclic subcover $Y \rightarrow Z$ of $f$ is given (birationally) by an equation of the form $y^{p}=u$, where $u$ is a certain rational function on $Z$, unique up to multiplication with a $p$ th power. We may assume that the $K$-model $Y_{K} \rightarrow Z_{K}$ (obtained from the stable model of $f$ ) is defined by the same equation. By assumption, $Y_{K} \rightarrow Z_{K}$ extends to a finite map $Y_{R} \rightarrow Z_{R}$ between semistable $R$-curves, where $Y_{R}$ is the stably pointed model of $Y_{K}$ and $Z_{R}=Y_{R} /(\mathbb{Z} / p)$. Neglecting the $\mathbb{Z} / m$-action on $Z_{R}$, this is precisely the situation studied in [14] (see also [6] and [8]).

Choose a vertex $v \in V$, and write $\bar{Y}_{v}$ for the inverse image of $\bar{Z}_{v}$ in $\bar{Y}$. We distinguish the following three cases (compare with [14], §1.4 and [8], §5.1):

- (multiplicative reduction) Suppose that the map $\bar{Y}_{v} \rightarrow \bar{Z}_{v}$ is inseparable (therefore, $v \in V^{\prime}$ is an interior vertex, by Assumption 2.2). Suppose, moreover, that the restriction $\bar{u}_{v}:=\left.u\right|_{\bar{Z}_{v}}$ of $u$ to $\bar{Z}_{v}$ is not a $p$ th power (in the function field of $\bar{Z}_{v}$ ). Then the cover $\bar{Y}_{v} \rightarrow \bar{Z}_{v}$ is given (birationally) by the equation $y^{p}=\bar{u}_{v}$ and carries a natural structure of $\boldsymbol{\mu}_{p}$-torsor over the open subset $\bar{U}_{v}:=\bar{Z}_{v}-\bar{g}^{-1}\left(\left\{\bar{x}_{e} \mid s(e)=v\right\}\right)$. Let $\omega_{v}:=d \bar{u}_{v} / \bar{u}_{v}$. The differential $\omega_{v}$ is not zero, regular on $\bar{Z}_{v}$ and does not depend on the choice of the rational function $u$. One easily checks that $\tilde{\beta}^{*} \omega_{v}=\bar{\chi}(1) \omega_{v}$. We write $\omega_{v} \in H^{0}\left(\bar{Z}_{v}, \Omega^{1}\right)_{\bar{\chi}}$. Furthermore, $\omega_{v}$ has no zero on $\bar{U}_{v}$.

- (additive reduction) Suppose that $\bar{Y}_{v} \rightarrow \bar{Z}_{v}$ is inseparable (hence $v \in V^{\prime}$ ), and that the restriction of $u$ to $\bar{Z}_{v}$ is a $p$ th power. Then, in a neighborhood of any point $\bar{z} \in \bar{Z}_{v}$ on $Z_{R}$ and after multiplying $u$ with a suitable $p$ th power, we can write $u=1+\pi^{p} w_{v}$, such that $\pi \in R$, $0<v_{R}(\pi)<v_{R}(p) /(p-1)$ and such that $\bar{w}_{v}:=\left.w_{v}\right|_{\bar{Z}_{v}}$ is not a $p$ th power. The restriction of $\bar{Y}_{v} \rightarrow \bar{Z}_{v}$ to the open subset $\bar{U}_{v}:=\bar{Z}_{v}-\bar{g}^{-1}\left(\left\{\bar{x}_{e} \mid s(e)=v\right\}\right)$ carries a natural structure of $\boldsymbol{\alpha}_{p}$-torsor, locally given by the equation $\tilde{y}^{p}=\bar{w}_{v}$. The differential $\omega_{v}:=d \bar{w}_{v}$ is not zero, independent of all the choices we have made, and is regular on $\bar{U}_{v}$. Again, one easily checks that $\tilde{\beta}^{*} \omega_{v}=\bar{\chi}(1) \omega_{v}$, i.e. $\omega_{v} \in H^{0}\left(\bar{U}_{v}, \Omega^{1}\right)_{\bar{\chi}}$. Furthermore, $\omega_{v}$ has no zero on $\bar{U}_{v}$.

- (étale reduction) If $v$ is a leaf, then $\bar{Y}_{v} \rightarrow \bar{Z}_{v}$ is generically étale, ramified only at $\bar{g}_{v}^{-1}\left(\bar{x}_{e}\right)$, where $e$ is the unique edge with $s(e)=v$. Choose a point $\bar{z} \in \bar{Z}_{v}$ above $\bar{x}_{e} \in \bar{X}_{v}$. In a neighborhood of $\bar{z}$, the cover $\bar{Y}_{v} \rightarrow \bar{Z}_{v}$ is an Artin-Schreier cover, with equation $y^{p}-y=w^{-h_{v}}$, where $w$ is some local coordinate for $\bar{Z}_{v}$ at $\bar{z}$ and $h_{v}$ is the conductor.

We shall say that the vertex $v$ has multiplicative, additive or étale reduction, according to which of the three cases occurs.

The data $\left(\omega_{v}, h_{v}\right)$ which we obtained from the reduction of the étale $p$-cyclic cover $Y \rightarrow Z$ satisfies certain compatibility conditions, see [14]. In our situation, they can be formulated as follows. Let $e$ be an edge, and let $\bar{z}_{e} \in \bar{Z}$ be a point above $\bar{x}_{e} \in \bar{X}$. Define

$$
h_{e}:= \begin{cases}\operatorname{ord}_{\bar{z}_{e}}\left(\omega_{v}\right)+1 & \text { if } v:=s(e) \in V^{\prime} \\ -h_{v} & \text { if } v:=s(e) \in B\end{cases}
$$


Since $\omega$ is an eigenvector under the $\mathbb{Z} / m$-action, $h_{e}$ is well defined. As a special case of [14], Cor. 2.8, we obtain

$$
h_{e}+h_{\bar{e}}=0,
$$

for all edges $e$. (It is also possible to derive (13) from [8], Proposition 5.2.1.)

Using the fact that the data $\left(\omega_{v} ; h_{i}\right)$ has to be compatible with the $\mathbb{Z} / m$-action, we can express the numbers $h_{e}$ in terms of certain (more convenient) numbers $\nu_{e}$. This generalizes Proposition 1.8.

PROPOSITION 2.5. - For each edge e, there exists an integer $\nu_{e}$ such that

$$
h_{e}=\frac{\nu_{e} m+a_{e}}{\left(a_{e}, m\right)} .
$$

The collection $\left(\nu_{e}\right)$ satisfies

$$
\nu_{e}+\nu_{\bar{e}}=-1
$$

and

$$
\sum_{s(e)=v}\left(\nu_{e}-1\right)=-3
$$

for all interior vertices $v$.

Proof.-Recall that $\tilde{\beta}^{a_{e}}$ is the canonical generator of inertia for the $m$-cyclic cover $\bar{g}_{v}: \bar{Z}_{v} \rightarrow \bar{X}_{v}$ above $\bar{x}_{e}$, see Proposition 2.4. If $a_{e} \in\{0, m\}$ then $\left(a_{e}, m\right)=m$, and the existence of an integer $\nu_{e}$ as in (14) is trivial. Now assume $0<a_{e}<m$, i.e. $\bar{Z}_{v} \rightarrow \bar{X}_{v}$ is actually branched at $\bar{x}_{e}$, of order $m /\left(a_{e}, m\right)$. Then the vertex $s(e)$ is either an interior vertex, or an element of $I$. Suppose that $v:=s(e)$ is an interior vertex. The equality $\left(\tilde{\beta}^{a}\right)^{*} \omega_{v}=\bar{\chi}(a) \omega_{v}$ implies the congruence $\operatorname{ord}_{\bar{z}_{e}}\left(\omega_{v}\right) \equiv a_{e} /\left(a_{e}, m\right)-1 \bmod m /\left(a_{e}, m\right)$. This proves the existence of $\nu_{e}$ in this case. The case $i=s(e) \in I$ has already been proved in Proposition 1.8. This completes the proof of the existence of the integers $\nu_{e}$. Now (15) follows from (13), (14) and the equality $a_{e}+a_{\bar{e}}=m$ by a straightforward calculation. Finally, (16) is a direct consequence of Proposition 2.4, Eq. (14) and the Riemann-Hurwitz formula.

We shall call an edge e terminal if $v:=t(e)$ is a leaf; for such an edge,

$$
h_{v}=h_{e}=\left(\nu_{e} m+a_{e}\right) /\left(a_{e}, m\right)
$$

is the conductor of the Artin-Schreier cover $\bar{Y}_{v} \rightarrow \bar{Z}_{v}$. In particular,

$$
h_{v}=\nu_{e} \quad \text { if } v=t(e) \in B-I .
$$

If $f$ is special, then $I=B$; moreover, for each terminal edge $e$ (with $i:=t(e) \in I$ ), the integer $\nu_{e}=\nu_{i}$ is either 0 or 1 and takes the value 0 for exactly three terminal edges (see Definition 1.9). In this case, we can also say a lot about the values $\nu_{e}$ on all edges $e$.

LeMma 2.6. - Assume that $f$ is special. Then:

(i) The integers $\nu_{e}$ (defined in Proposition 2.5) lie between -2 and 1.

(ii) There exists a unique interior vertex $v_{0} \in V^{\prime}$ such that $\nu_{e} \geqslant 0$ for all edges e with source $v_{0}$.

(iii) If $v \neq v_{0}$ is an interior vertex, then there exists a unique edge e with source $v$ such that $\nu_{e}<0$. 
Proof.-Since $f$ is special, we may assume that $\nu_{i}=0$ for $i=1,2,3$ and $\nu_{i}=1$ for $i=4, \ldots, r$. Furthermore, $I=B$. For any edge $e \in E$, let $I_{e} \subset I$ be the set of leaves $i \in I$ which 'lie in the direction of $e$ '. More precisely, $i \in I_{e}$ if and only if $i$ lies in the connected component of $T-\{e\}$ which contains the vertex $t(e)$. We claim that

$$
\nu_{e}=1-\left|I_{e} \cap\{1,2,3\}\right|,
$$

for all $e \in E$. Let us check that the lemma follows from this claim. Indeed, (i) is a trivial consequence of (17), and parts (ii) and (iii) of the lemma follow once we observe that $v_{0} \in V$ has to be the median of the three leaves $i=1,2,3$.

Recall that $\nu_{e}=\nu_{i}$ if $i:=t(e) \in I$. Using $\nu_{i}=0$ for $i=1,2,3$ and $\nu_{i}=1$ for $i>3$, we conclude that (17) holds for all edges $e$ such that $t(e) \in I$. For a general edge $e \in E$, define $\nu_{e}^{\prime}:=1-\left|I_{e} \cap\{1,2,3\}\right|$. An easy verification shows that the function $e \mapsto \nu_{e}^{\prime}$ verifies Eqs. (15) and (16). We conclude that $\nu_{e}=\nu_{e}^{\prime}$ for all edges $e \in E$, by induction. This finishes the proof of the lemma.

Remark 2.7. - It is shown in [8] that the integers $h_{e}$ determine the radii of the formal annuli corresponding to the singular points of $Y_{R}$. To be more precise, let $\bar{y}$ be an ordinary double point of the special fiber of a semistable $R$-curve $Y_{R}$. Then the complete local ring of $Y_{R}$ at $\bar{y}$ is of the form $\hat{\mathcal{O}}_{Y_{R}, \bar{y}} \cong R[[u, v \mid u v=\pi]]$, with $\pi \in R$. We define the thickness of $Y_{R}$ at $\bar{y}$ as the (positive rational) number $\varepsilon\left(Y_{R}, \bar{y}\right):=v_{R}(\pi)$ (recall that $v_{R}(p)=1$ ). Suppose $\bar{y} \in \bar{Y}$ is a point above $\bar{x}_{e} \in \bar{X}, e \in E$. Suppose, moreover, that $v:=s(e)$ is a vertex with multiplicative reduction and $v^{\prime}:=t(e)$ has additive reduction. Then

$$
0<\varepsilon\left(Y_{R}, \bar{y}\right)<\frac{1}{(p-1) h_{e}}
$$

On the other hand, if $v:=s(e)$ has multiplicative and $v^{\prime}:=t(e)$ étale reduction, then

$$
\varepsilon\left(Y_{R}, \bar{y}\right)=\frac{1}{(p-1) h_{e}} .
$$

This follows immediately from [8], Chap. 5, Proposition 2.1. Moreover, using [13], Proposition 2.3.2, one shows that

$$
\varepsilon\left(X_{R}, \bar{x}_{e}\right)=\frac{p a_{e}}{\left(a_{e}, m\right)} \cdot \varepsilon\left(Y_{R}, \bar{y}\right)
$$

\subsection{The proof of Theorem 2.1}

Let $v_{0}$ be the 'median vertex' of Lemma 2.6(ii). Theorem 2.1 is equivalent to the statement that $v_{0}$ is the unique interior vertex. Therefore, let us assume that there exists another interior vertex $v \neq v_{0}$, and then try to arrive at a contradiction. By Lemma 2.6(iii), there exists a unique edge $e$ with $s(e)=v$ such that $\nu_{e}<0$. This means that the differential $\omega_{v}$ has a pole in each point $\bar{z}_{e} \in \bar{Z}_{v}$ above $\bar{x}_{e} \in \bar{X}_{v}$. If the cover $Y \rightarrow Z$ had multiplicative reduction at the component $\bar{Z}_{v}$, then the differential $\omega_{v}$ would be regular on $\bar{Z}_{v}$. Therefore, we have additive reduction at $\bar{Z}_{v}$. In particular, the differential $\omega_{v}$ is a nonzero exact differential, i.e. of the form $\omega_{v}=d u$, for some rational function $u$ on $\bar{Z}_{v}$. Moreover, the divisor $\left(\omega_{v}\right)$ is completely determined by the integers $\nu_{e}$, where $e$ runs through the set of edges with source $v$. By Lemma 2.6(i) and Proposition 2.5 , these numbers satisfy $-2 \leqslant \nu_{e} \leqslant 1$ and $\sum_{s(e)=v}\left(\nu_{e}-1\right)=-3$. The following lemma gives the desired contradiction, and thus finishes the proof of Theorem 2.1. 
LEMMA 2.8. - Let $k$ be an algebraically closed field of characteristic $p>0, m>1$ an integer dividing $p-1$, and $r \geqslant 3$. Let $g: Z \rightarrow \mathbb{P}_{k}^{1}$ be an $m$-cyclic cover given (birationally) by an equation of the form

$$
z^{m}=\prod_{i=1}^{r}\left(x-x_{i}\right)^{a_{i}},
$$

with $x_{1}, \ldots, x_{r} \in k$ pairwise distinct, and $0<a_{i}<m$ such that $\sum_{i} a_{i}=m$. Let $\phi: Z \stackrel{\sim}{\rightarrow} Z$ be $a$ generator of $\operatorname{Aut}\left(Z / \mathbb{P}_{k}^{1}\right)$, such that $\phi^{*} z=\zeta z, \zeta \in k^{\times}$an mth root of unity. For $i=1, \ldots, m$, let $m_{i}:=m /\left(a_{i}, m\right), \tilde{a}_{i}:=a_{i} /\left(a_{i}, m\right)$ and $P_{i}:=g^{-1}\left(x_{i}\right)$ (considered as a divisor on $\left.Z\right)$. Let $\omega$ be a meromorphic differential form on $Z$ such that

(i) $\phi^{*} \omega=\zeta \omega$, and

(ii) $(\omega)=\sum_{i=1}^{r}\left(m_{i} \nu_{i}+\tilde{a}_{i}-1\right) P_{i}$, with integers $-2 \leqslant \nu_{i} \leqslant 1$ such that $\sum_{i} \nu_{i}=r-3, \nu_{1}<0$ and $\nu_{i} \geqslant 0$ for $i>1$.

Then $\mathcal{C}(\omega) \neq 0$, i.e. $\omega$ is not exact.

Proof. - Assume that $\omega$ is a meromorphic differential on $Z$ such that (i) and (ii) hold. After a change of coordinate, we may assume that $x_{1}=0$. By (i), we can write $\omega=f z \mathrm{~d} x$, where $f$ is a rational function in $x$. Expanding $f$ as a Taylor series at $x=x_{1}=0$, we obtain

$$
\omega=\left(\sum_{j=-3}^{\infty} c_{j} x^{j}\right) z \mathrm{~d} x,
$$

with $c_{j} \in k$. Note that $x$ (resp. $z$ ) has a zero of order $m_{1}$ (resp. of order $\tilde{a}_{1}$ ) at each point $\bar{z} \in P_{1}$. In particular, the coefficients $c_{-3}$ and $c_{-2}$ contribute to the poles of $\omega$ in $P_{1}$, which are of order $\left(-m_{1} \nu_{1}+a_{1}-1\right)$, by (ii).

Claim 1. - There exist elements $b_{1}, b_{2} \in k$ such that

$$
\omega^{\prime}:=\omega-\mathrm{d} u \text { is regular on } Z \text {, where } u:=\left(b_{1} x^{-2}+b_{2} x^{-1}\right) z .
$$

Since $\omega$ and $u$ are regular away from $P_{1}$, we only have to pay attention to the points in $P_{1}$. We compute 'Taylor series' as in (21):

$$
\mathrm{d} z=\left(\frac{a_{1}}{m} x^{-1}+d_{0}+d_{1} x+\cdots\right) z \mathrm{~d} x
$$

and

$$
\begin{aligned}
\mathrm{d} u & =\left(-2 b_{1} x^{-3}-b_{2} x^{-2}\right) z \mathrm{~d} x+\left(b_{1} x^{-2}+b_{2}, x^{-1}\right) \mathrm{d} z \\
& =\left(\left(\frac{a_{1}}{m}-2\right) b_{1} x^{-3}+\left(d_{0} b_{1}+\left(\frac{a_{1}}{m}-1\right) b_{2}\right) x^{-2}+\cdots\right) z \mathrm{~d} x .
\end{aligned}
$$

Hence, to prove Claim 1 , we have to find $b_{1}, b_{2}$ such that

$$
\left(\frac{a_{1}}{m}-2\right) b_{1}=c_{-3}, \quad d_{0} b_{1}+\left(\frac{a_{1}}{m}-1\right) b_{2}=c_{-2} .
$$

Using $m \mid p-1$ and $a_{1} \leqslant m-2$ one shows that $p$ does not divide $2 m-a_{1}$ and $m-a_{1}$; therefore, $a_{1} / m-2, a_{1} / m-1 \neq 0$ in $k$, and we can solve (25) in $b_{1}$ and $b_{2}$. This proves Claim 1. 
Claim 2. $-\omega \neq \mathrm{d} u$.

Assuming the contrary, we would have

$$
(\mathrm{d} u)=(\omega)=\sum_{i=1}^{r}\left(m_{i} \nu_{i}+\tilde{a}_{i}-1\right) P_{i},
$$

by condition (ii). Assume for the moment that the order of $u$ at all the ramification points is prime-to- $p$. Then (26) implies

$$
(u) \geqslant \sum_{i}\left(m_{i} \nu_{i}+\tilde{a}_{i}\right) P_{i} .
$$

But the divisor on the right has degree $\sum_{i} m \nu_{i}+a_{i}=m(r-2)>0$, contradiction! Thus, in order to prove Claim 2, it suffices to show that $u$ has no zero or pole in one of the ramification points of order divisible by $p$. Let $z_{i} \in P_{i}$ be a ramification point above $x_{i}$, for some $i$. Since $\phi^{*} u=\zeta u, \operatorname{ord}_{z_{i}}(u)=m_{i} k+\tilde{a}_{i}$, for some integer $k$. If $p$ divides $\operatorname{ord}_{z_{i}}(u)=m_{i} k+\tilde{a}_{i}$ then either $k \geqslant 1$ or $k<0$. In the first case, we would have $\operatorname{ord}_{z_{i}}(\mathrm{~d} u)=m_{i} \nu_{i}+\tilde{a}_{i}>m_{i}+\tilde{a}_{i}$, hence $\nu_{i}>1$, which contradicts our assumptions. The second case can occur only for $i=1$. But for $i=1, k \in\{-2,-1\}$, and in this case we have already shown that $k m_{i}+\tilde{a}_{i}$ is prime to $p$ (see the end of the proof of Claim 1). We conclude that $\omega \neq \mathrm{d} u$, as asserted by Claim 2 .

Set $\omega^{\prime}:=\omega-\mathrm{d} u$, and note that $\phi^{*} \omega^{\prime}=\zeta \omega^{\prime}$, by condition (i) and the definition of $u$. Following our previous notation, we can write $\omega^{\prime} \in H^{0}\left(Z, \Omega_{Z / k}^{1}\right)_{\chi}$, where $\chi: \mathbb{Z} / m \rightarrow k^{\times}$is the character with $\chi(1)=\zeta$. By Lemma 1.4(iv), we have $\mathcal{C}(\omega)=\mathcal{C}\left(\omega^{\prime}\right) \neq 0$. This proves the lemma, and also Theorem 2.1.

\subsection{The monodromy group of a special cover}

The analysis of the stable reduction of a special cover shows somewhat more than what is stated in Theorem 2.1. We use the same notation as in Theorem 2.1. In particular, $f: Y \rightarrow \mathbb{P}^{1}$ is a special cover of type $\left(x_{i} ; a_{i}\right)$, with stable reduction $\bar{f}: \bar{Y} \rightarrow \bar{X}$. Define

$$
\mathcal{D}_{i}=\left\{x \in \mathbb{P}^{1}(\bar{K}) \mid x \text { specializes to a point on } \bar{X}_{i}-\bar{X}_{0} \cong \mathbb{A}_{k}^{1}\right\},
$$

the closed rigid disk containing all points of $\mathbb{P}^{1}$ which specialize to $\bar{X}_{i}$. In particular, $x_{i} \in \mathcal{D}_{i}$.

Proposition 2.9. - We have

$$
\mathcal{D}_{i}=\left\{x \in \mathbb{P}^{1}(\bar{K}) \mid v_{R}\left(x-x_{i}\right) \geqslant \frac{p m_{i}}{(p-1) h_{i}}\right\} .
$$

(Recall that $m_{i}=m /\left(a_{i}, m\right)$ and $h_{i}=\left(m \nu_{i}+a_{i}\right) /\left(a_{i}, m\right)$.)

Proof. - This follows immediately from Remark 2.7, Eqs. (19) and (20).

For the rest of this section, we assume that the absolute ramification index of $K_{0}$ is one. Thus, we can identify $R_{0}$ with the ring $W(k)$ of Witt vectors over the residue field $k$ (in view of the results of Section 3, this is not a serious restriction). By Proposition 1.3, the field of moduli of $f$ is $K^{\text {in }}=K_{0}\left(\zeta_{p}^{(m)}\right)$. Let $K=K^{\text {st }}$ be the minimal extension of $K^{\text {in }}$ over which $f$ has stable reduction. It follows from [13] that the extension $K / K^{\text {in }}$ is Galois, of degree prime-to- $p$. The Galois group $\Gamma:=\operatorname{Gal}\left(K / K^{\text {in }}\right)$ acts faithfully and $k$-linearly on $\bar{Y}$ (where $\bar{f}: \bar{Y} \rightarrow \bar{X}$ is the stable reduction of $f$ ), and this action commutes with the action of $H$. Therefore, we get an induced action of $\Gamma$ on $\bar{X}$. The group $\Gamma$ is called the monodromy group of $f$. 
THEOREM 2.10. - Let $f: Y \rightarrow \mathbb{P}^{1}$ be a special cover of type $\left(x_{i} ; a_{i}\right)$. Assume that the branch points $x_{i}$ are rational over $K_{0}$, the fraction field of $W(k)$. Then the order of the monodromy group $\Gamma$ of $f$ is

$$
|\Gamma|=\left[K: K^{\mathrm{in}}\right]=m \cdot \operatorname{lcm}\left(h_{1}, \ldots, h_{r}\right) .
$$

Furthermore, the action of $\Gamma$ is trivial on $\bar{X}_{0}$ and cyclic of order $h_{i}(p-1) / m_{i}$ on $\bar{X}_{i}$.

Proof. - The proof of Theorem 2.1 shows that there exists an open subset $U \subset Z_{R}$ such that $U \otimes_{R} k \subset \bar{Z}_{0}$ is nonempty and $V:=U \times_{Z_{R}} Y_{R} \rightarrow U$ is a $\mu_{p}$-torsor. But the generic fiber $V_{K} \rightarrow U_{K}$ is a $\mathbb{Z} / p$-cover. Therefore, the extension $K / K_{0}$ contains the $p$ th roots of unity. Moreover, the subgroup $\widetilde{\Gamma}:=\operatorname{Gal}\left(K / K_{0}\left(\zeta_{p}\right)\right) \subset \Gamma$ is precisely the stabilizer of $\bar{Y}_{0} \subset \bar{Y}$ (where $\bar{Y}_{0}$ denotes the inverse image of $\bar{Z}_{0}$ ). It follows from Proposition 1.3(ii) that $\Gamma / \widetilde{\Gamma} \cong \mathbb{Z} / m$. Recall that $\bar{Y}$ is the union of $\bar{Y}_{0}$ and $\bar{Y}_{i}$, the (possibly disconnected) inverse image of $\bar{X}_{i}$, for $i=1, \ldots, r$. Let $\bar{y}$ be a point where $\bar{Y}_{i}$ intersects $\bar{Y}_{0}$, and let $\bar{Y}_{i}^{\prime}$ be the connected component of $\bar{Y}_{i}$ containing $\bar{y}$. Since $\widetilde{\Gamma}$ acts trivially on $\bar{Y}_{0}$, it fixes $\bar{Y}_{i}^{\prime}$ and $\bar{y}$. By Remark 2.7, Eq. (20), $\varepsilon\left(Y_{R}, \bar{y}\right)=1 /(p-1) h_{i}$. This means that the complete local ring of $Y_{R}$ at $\bar{y}$ is of the form $R\left[\left[u, v \mid u v=\lambda^{1 / h_{i}}\right]\right]$, with $\lambda:=\zeta_{p}-1 \in K_{0}\left(\zeta_{p}\right)$. Applying an element of $\widetilde{\Gamma}$ to the equation $u v=\lambda^{1 / h_{i}}$, one shows that $\widetilde{\Gamma}$ induces a cyclic action on $\bar{Y}_{i}^{\prime}$, of order $h_{i}$. More precisely, the image of $\widetilde{\Gamma}$ in $\operatorname{Aut}_{k}\left(\bar{Y}_{i}^{\prime}\right)$ is the quotient $\operatorname{Gal}\left(K_{0}\left(\zeta_{p}, \lambda^{1 / h_{i}}\right) / K_{0}\left(\zeta_{p}\right)\right) \cong \mathbb{Z} / h_{i}$. Note that the action of $\widetilde{\Gamma}$ on $\bar{Y}_{i}^{\prime}$ commutes with the action of the decomposition group $H_{i} \subset H$ of $\bar{Y}_{i}^{\prime}$, which is of order $p m_{i}$. Now the statement of Theorem 2.10 on the order of $\Gamma$ follows from the fact that the action of $\Gamma$ on $\bar{Y}$ is faithful. The statement about the action of $\Gamma$ on $\bar{X}_{i}$ follows as well, using the fact that $h_{i}$ is relatively prime to $\mathrm{pm}_{i}$ (it can also be deduced directly from Proposition 2.9).

\section{Construction of special covers}

This section is concerned with the construction of special covers by lifting certain objects from characteristic $p$ to characteristic 0 . We start by defining special degeneration data, which are essentially given by an $m$-cyclic cover $\bar{Z}_{0} \rightarrow \mathbb{P}_{k}^{1}$ of the projective line in characteristic $p$, together with a logarithmic differential form $\omega_{0}$ on $\bar{Z}_{0}$, with certain prescribed zeros. It is immediate from the results of the previous section that the reduction $\bar{f}: \bar{Y} \rightarrow \bar{X}$ of a special cover $f$ corresponds essentially to a special degeneration datum. The main result of this section (Theorem 3.2) states that, conversely, every special degeneration datum arises as the reduction of a special cover $f$. Moreover, the cover $f$ is essentially unique, once we have chosen the branch points $x_{i}$.

The proof of Theorem 3.2 is divided into two steps. In the first step, we lift the $\boldsymbol{\mu}_{p}$-torsor $\bar{Y}_{0} \rightarrow \bar{Z}_{0}$ corresponding to the differential $\omega_{0}$ to characteristic 0 , in a $\mathbb{Z} / m$-equivariant way. This construction yields a metacyclic cover $f: Y \rightarrow \mathbb{P}^{1}$, which is essentially unique because the $\bar{\chi}$-eigenspace of the $p$-torsion of the Jacobian of $\bar{Z}_{0}$ is étale (Proposition 1.3). In the second step we show that the cover $f$ we have constructed is special provided that we have chosen the branch points $x_{i}$ inside certain closed rigid disks $\mathcal{D}_{i} \subset \mathbb{P}^{1}$. The proof uses the monodromy action on the stable reduction of Galois covers, and a deformation argument.

In Section 3.5 we determine all special degeneration data in the case $r=4$.

\subsection{Special degeneration data}

Let $f: Y \rightarrow \mathbb{P}^{1}$ be a special cover of type $\left(x_{i} ; a_{i}\right)$, with stable model $f_{R}: Y_{R} \rightarrow X_{R}$ and reduction $\bar{f}: \bar{Y} \rightarrow \bar{X}$ (see Definition 1.9). By Theorem 2.1, $\bar{X}$ consists of $r+1$ components $\bar{X}_{0}, \ldots, \bar{X}_{r}$, such that, for $i \geqslant 1, \bar{X}_{i}$ is the tail containing the specialization $\bar{x}_{i}$ of the branch 
point $x_{i}$. The component $\bar{X}_{0}$, which intersects all of the components $\bar{X}_{i}, i \geqslant 1$, is called the original component of $\bar{X}$. We have a canonical isomorphism $\bar{X}_{0} \cong \mathbb{P}_{k}^{1}$ arising from the contraction morphism $q: X_{R} \rightarrow \mathbb{P}_{R}^{1}$. This isomorphism identifies the intersection point $\tau_{i} \in \bar{X}_{0} \cap \bar{X}_{i}$ with the specialization of $x_{i}$, regarded as point on $\mathbb{P}_{R}^{1}$. We may assume, without loss of generality, that $\tau_{i} \neq \infty$.

We have seen in Section 2.1 that the map $\bar{f}: \bar{Y} \rightarrow \bar{X}$ is the composition of an $m$-cyclic admissible cover $\bar{g}: \bar{Z} \rightarrow \bar{X}$ with a finite map $\bar{Y} \rightarrow \bar{Z}$ of degree $p$ which is the reduction of the étale $p$-cyclic cover $Y \rightarrow Z$. By Proposition 2.4, the restriction $\bar{g}_{0}: \bar{Z}_{0} \rightarrow \bar{X}_{0}=\mathbb{P}_{k}^{1}$ of $\bar{g}$ to the original component can be identified with the $m$-cyclic cover of type $\left(\tau_{i} ; a_{i}\right)$. Moreover, the $p$-cyclic cover $Y \rightarrow Z$ has multiplicative reduction at $\bar{Z}_{0}$. This means that the induced cover $\bar{Y}_{0} \rightarrow \bar{Z}_{0}$ carries the structure of a $\boldsymbol{\mu}_{p}$-torsor. This structure gives rise to a regular differential form $\omega_{0}$ such that $\beta^{*} \omega_{0}=\bar{\chi}(1) \omega_{0}$. As before, we write $\omega_{0} \in H^{0}\left(\bar{Z}_{0}, \Omega_{1}\right)_{\bar{\chi}}$. Let $m_{i}:=m /\left(a_{i}, m\right)$, $\tilde{a}_{i}:=a_{i} /\left(a_{i}, m\right)$ and $P_{i}:=\bar{g}_{0}^{-1}\left(\tau_{i}\right)$ (we regard $P_{i}$ as a divisor on $\bar{Z}_{0}$ ). By Proposition 2.5, there exist integers $\nu_{i} \in\{0,1\}$ with $\sum_{i} \nu_{i}=r-3$ such that

$$
\left(\omega_{0}\right)=\sum_{i}\left(m_{i} \nu_{i}+\tilde{a}_{i}-1\right) P_{i}
$$

Furthermore, $\omega_{0}$ is logarithmic. In terms of the Cartier operator $\mathcal{C}$, this means that

$$
\mathcal{C}\left(\omega_{0}\right)=\omega_{0}
$$

Definition 3.1. - Let $k$ be an algebraically closed field of characteristic $p>0$. A special degeneration datum over $k$ is given by

- pairwise distinct $k$-rational points $\tau_{1}, \ldots, \tau_{r} \in \mathbb{P}_{k}^{1}$, with $r \geqslant 3$,

- an integer $m>1$ dividing $p-1$, and integers $a_{1}, \ldots, a_{r}$ such that $0<a_{i}<m$ and $\sum_{i} a_{i}=m$, (we let $\bar{g}_{0}: \bar{Z}_{0} \rightarrow \mathbb{P}_{k}^{1}$ be the $m$-cyclic cover of type $\left(\tau_{i} ; a_{i}\right)$; furthermore, we set $m_{i}:=m /\left(a_{i}, m\right), \tilde{a}_{i}:=a_{i} /\left(a_{i}, m\right)$ and $\left.P_{i}:=\bar{g}_{0}^{-1}\left(\tau_{i}\right)\right)$,

- integers $\nu_{1}, \ldots, \nu_{r} \in\{0,1\}$ such that $\sum_{i} \nu_{i}=r-3$, and

- a differential form $\omega_{0} \in H^{0}\left(\bar{Z}_{0}, \Omega^{1}\right)_{\bar{\chi}}$, such that (29) and (30) hold.

As explained in the paragraph preceding Definition 3.1, we can attach to (the reduction of) a special cover $f: Y \rightarrow \mathbb{P}^{1}$ of type $\left(x_{i} ; a_{i}\right)$ a special degeneration datum $\left(\tau_{i} ; a_{i} ; \nu_{i} ; \omega_{0}\right)$. Theorem 3.2 below states that, conversely, every special degeneration datum arises in this way.

For the rest of this section, we fix a special degeneration datum $\left(\tau_{i} ; a_{i} ; \nu_{i} ; \omega_{0}\right)$ over $k$. Let $K_{0}$ denote the fraction field of $R_{0}:=W(k)$, the ring of Witt vectors over $k$. Choose an algebraic closure $\bar{K}$ of $K_{0}$. For $i \in I$, choose a $K_{0}$-rational point $\tilde{x}_{i} \in \mathbb{P}^{1}\left(K_{0}\right)$ which lifts $\tau_{i} \in \mathbb{P}^{1}(k)$. Let

$$
\mathcal{D}_{i}:=\left\{x \in \mathbb{P}^{1}(\bar{K}) \mid v_{R}\left(x-\tilde{x}_{i}\right) \geqslant \frac{p m_{i}}{(p-1) h_{i}}\right\}
$$

(compare to the statement of Theorem 2.10). We claim that the collection of disks $\left(\mathcal{D}_{i}\right)$ does not depend, up to an automorphism of $\mathbb{P}_{K_{0}}^{1}$, on the choice of the points $\tilde{x}_{i}$. To show that this is so, we may assume that $\nu_{1}=\nu_{2}=\nu_{3}=0$. Furthermore, we can always normalize our choice such that $\tilde{x}_{1}=0, \tilde{x}_{2}=1$ and $\tilde{x}_{3}=\infty$. For $i=4, \ldots, r$, we have $0<p m_{i} /(p-1) h_{i}<1$. Using the triangle inequality and the fact that the valuation $v_{R}$ takes integral values on $K_{0}$, one shows that $\mathcal{D}_{i}$ does not depend on the choice of $\tilde{x}_{i}$, for $i=4, \ldots, r$.

THEOREM 3.2. - Let $\left(\tau_{i} ; a_{i} ; \nu_{i} ; \omega_{0}\right)$ be a special degeneration datum over $k$ and let $x_{1}, \ldots, x_{r}$ be $\bar{K}$-rational points on $\mathbb{P}^{1}$, such that $x_{i} \in \mathcal{D}_{i}$. Then there exists a special cover $f: Y \rightarrow \mathbb{P}^{1}$ of type $\left(x_{i} ; a_{i}\right)$, unique up to isomorphism, which gives rise to $\left(\tau_{i} ; a_{i} ; \nu_{i} ; \omega_{0}\right)$. 
This theorem implies Theorem B in the introduction. The proof is given in the next three subsections. We will prove in [21] that the condition $x_{i} \in \mathcal{D}_{i}$ is also necessary for $f$ to be special. Unfortunately, the method of proof we use here does not give such an 'if and only if' result.

\subsection{Definition of the lift}

Let $\left(\tau_{i} ; a_{i} ; \nu_{i} ; \omega_{0}\right)$ be a special degeneration datum over $k$ and $\bar{g}_{0}: \bar{Z}_{0} \rightarrow \mathbb{P}_{k}^{1}$ the $m$-cyclic cover of type $\left(\tau_{i} ; a_{i}\right)$. Choose $\bar{K}$-rational points $x_{i} \in \mathbb{P}^{1}(\bar{K})$ lifting the points $\tau_{i}$ (for the moment, we do not assume that $x_{i} \in \mathcal{D}_{i}$ ). Choose a finite extension $K / K_{0}$ such that $x_{i}$ is $K$-rational; let $R$ be the ring of integers of $K$.

The $m$-cyclic cover $\bar{g}_{0}$ lifts uniquely to an $m$-cyclic cover $g_{R}^{\prime}: Z_{R}^{\prime} \rightarrow \mathbb{P}_{R}^{1}$ of smooth curves, tamely ramified along the closure of $\left\{x_{1}, \ldots, x_{r}\right\} \subset \mathbb{P}_{K}^{1}$ inside $\mathbb{P}_{R}^{1}$. Let $g_{K}: Z_{K} \rightarrow \mathbb{P}_{K}^{1}$ be the generic fiber of $g_{R}^{\prime}$ and $g: Z \rightarrow \mathbb{P}^{1}$ its base change to $\bar{K}$. Note that $g$ is the $m$-cyclic cover of type $\left(x_{i} ; a_{i}\right)$. Let $J_{R}$ be the Néron-model of $J_{Z_{K}}$ over $R$. Since $Z_{R}^{\prime}$ is smooth over $R, J_{R}$ represents the functor $\operatorname{Pic}^{0}\left(Z_{R}^{\prime} / R\right)$, see [1], §9, Proposition 4 . The universal property of the Néron model defines a surjective specialization map $J_{Z_{K}}(K) \rightarrow J_{\bar{Z}_{0}}(k)$. By Proposition 1.3, the specialization map induces an isomorphism

$$
J_{Z}[p]_{\bar{\chi}} \stackrel{\sim}{\longrightarrow} J_{\bar{Z}_{0}}[p]_{\bar{\chi}}
$$

of $\mathbb{F}_{p}$-modules (of rank $r-2$ ).

The logarithmic differential $\omega_{0}$ corresponds to a line bundle $\bar{L}$ on $\bar{Z}_{0}$, in the following way (see e.g. [10], III, §4). Let $\bar{u}$ be a rational function on $\bar{Z}_{0}$ such that $\omega_{0}=d \bar{u} / \bar{u}$. Then $(\bar{u})=p \cdot \bar{D}$, for a divisor $\bar{D}$ of degree 0 on $\bar{Z}_{0}$; we set $\bar{L}:=\mathcal{O}_{\bar{Z}_{0}}(\bar{D})$. By definition, $\bar{L}{ }^{\otimes p} \cong \mathcal{O}_{\bar{Z}_{0}}$. Moreover, $\beta^{*} \omega_{0}=\bar{\chi}(1) \omega_{0}$ implies $\beta^{*} \bar{L} \cong \bar{L}^{\otimes \bar{\chi}(1)}$ (note that this makes sense because $\bar{\chi}(1) \in \mathbb{F}_{p}^{\times}$). In other words, $\bar{L}$ corresponds to an element of $J_{\bar{Z}_{0}}[p]_{\chi}$. Let $L$ be the line bundle on $Z$ corresponding to $\bar{L}$ under the isomorphism (32). By the definition of the specialization map (32), $L$ is actually the pullback of a line bundle $L_{R}$ on $Z_{R}^{\prime}$, and $L_{R} \cong \mathcal{O}_{Z_{R}^{\prime}}(D)$, where $D$ is a horizontal divisor on $Z_{R}^{\prime}$ such that $p \cdot D=(u)$ for some rational function $u$. By construction, we have $\omega_{0}=d \bar{u} / \bar{u}$, with $\bar{u}:=\left.u\right|_{\bar{Z}_{0}}$. We let $Y \rightarrow Z$ be the $\boldsymbol{\mu}_{p}$-torsor corresponding to $L$ (birationally given by the equation $y^{p}=u$ ). After choosing a $p$ th root of unity $\zeta_{p} \in \bar{K}$, we can regard $Y \rightarrow Z$ as an étale $p$-cyclic cover. Now the composition $f: Y \rightarrow Z \rightarrow \mathbb{P}^{1}$ is a metacyclic cover of type $\left(x_{i} ; a_{i}\right)$ (see Section 1.1).

The cover $f$ we have constructed will not be special, in general. However, if $f$ is special then, by construction, it gives rise to the special degeneration datum $\left(\tau_{i} ; a_{i} ; \nu_{i} ; \omega_{0}\right)$ we started with. It is also clear that any special cover which gives rise to $\left(\tau_{i} ; a_{i} ; \nu_{i} ; \omega_{0}\right)$ is isomorphic to $f$. Thus, in order to prove Theorem 3.2, we have to show that $f$ is special provided that $x_{i} \in \mathcal{D}_{i}$, for all $i \in I$. Before we can give a proof of this claim (in Section 3.4), we need to analyze the stable reduction of $f$. For this step, it is not yet necessary to assume $x_{i} \in \mathcal{D}_{i}$.

\subsection{Analyzing the stable reduction of $f$}

We may assume that the cover $f$ constructed above has stable reduction over the field $K$. Let $f_{R}: Y_{R} \rightarrow X_{R}$ be the stable model of $f$, and $\bar{f}: \bar{Y} \rightarrow \bar{X}$ its reduction. For $i=1, \ldots, r$, let $\bar{x}_{i} \in \bar{X}$ be the specialization of the branch point $x_{i}$, and let $\bar{X}_{i}$ be the component of $\bar{X}$ containing $\bar{x}_{i}$. Since $x_{i} \not \equiv x_{j} \bmod v_{R_{0}}$ for $i \neq j$ (as points on $\mathbb{P}^{1}$ ), it follows from [13], §3, that the components $\bar{X}_{i}$ are pairwise distinct tails of $\bar{X}$ and that $\bar{f}: \bar{Y} \rightarrow \bar{X}$ is separable exactly over the tails. In other words, Assumption 2.2 holds. Note that the stronger Assumption 1.6 may not hold, as $\bar{X}$ might have new tails.

However, Assumption 2.2 being valid, we may use the notation set up in Sections 2.1 and 2.2, concerning the structure of $\bar{f}: \bar{Y} \rightarrow \bar{X}$ as the composition of the $m$-cyclic admissible cover 
$\bar{g}: \bar{Z} \rightarrow \bar{X}$ and the 'mixed torsor' $\bar{Y} \rightarrow \bar{Z}$. Recall that we described this structure using certain combinatorial data $\left(T ; a_{e} ; \nu_{e}\right)$. Here $T$ is the dual graph of components of the semistable curve $\bar{X}$. The integers $a_{e}$ (where $e$ is an edge of $T$ ) describe the admissible $m$-cyclic cover $\bar{g}: \bar{Z} \rightarrow \bar{X}$. Finally, the integers $\nu_{e}$ (together with the $a_{e}$ ) determine the order of the zeros and the poles of the differentials $\omega_{v}$ attached to the torsors $\bar{Y}_{v} \rightarrow \bar{Z}_{v}$, where $v$ is an interior vertex of $T$. By construction of $f, Y \rightarrow Z$ has multiplicative reduction above the original component $\bar{X}_{0}$, and the resulting $\boldsymbol{\mu}_{p}$-torsor $\bar{Y}_{0} \rightarrow \bar{Z}_{0}$ corresponds to the differential $\omega_{0}$. Therefore, (29) implies that

$$
\nu_{e_{i}}=\nu_{i} \in\{0,1\}
$$

where $\nu_{i}$ is given by the special degeneration datum and $e_{i}$ is the edge corresponding to the point $\tau_{i} \in \bar{X}_{0}$ (in particular, $\tau_{i}$ is a singular point of $\bar{X}$ ). Note that we do not know (unless $f$ is special) whether $h_{i}=\nu_{i} m_{i}+\tilde{a}_{i}$ is the conductor of the Artin-Schreier cover $\bar{Y}_{i} \rightarrow \bar{Z}_{i}$ over the tail $\bar{X}_{i}$.

Let $v_{0} \in V$ be the vertex of the tree $T$ corresponding to the original component $\bar{X}_{0}$. For any edge $e$ of $T$, we let $T_{e}$ be the connected component of $T-\{e\}$ which contains $t(e)$. We shall call an edge $e$ positive if $v_{0} \notin T_{e}$, i.e. if $e$ is directed away from the vertex $v_{0}$.

Lemma 3.3. - (i) Let e be a positive edge. Then $\nu_{e} \geqslant 0$. If, moreover, $a_{e} \equiv 0 \bmod m$, then $\nu_{e}>1$.

(ii) The points $\tau_{1}, \ldots, \tau_{r}$ are precisely the points of $\bar{X}_{0}$ which are singular points of $\bar{X}$.

(iii) Fix $i \in I=\{1, \ldots, r\}$, and let $e_{i}$ be the edge with source $v_{0}$ corresponding to $\tau_{i}$. If $\nu_{i}=0$, then $T_{e_{i}}=\{i\}$. On the other hand, if $\nu_{i}=1$, then either $T_{e_{i}}=\{i\}$ or we are in the following case. The vertex $v:=t\left(e_{i}\right)$ is the source of exactly three edges, $\bar{e}_{i}, e^{\prime}, e^{\prime \prime}$. Also, $t\left(e^{\prime}\right)=i \in I$ and $t\left(e^{\prime \prime}\right) \in B-I$ is a leaf. See Fig. 1.

Proof. - Suppose we have a positive edge $e$ such that $a_{e} \equiv 0 \bmod m$ and $\nu_{e} \leqslant 1$. Note that this implies $a_{e^{\prime}} \equiv 0 \bmod m$ for all edges contained in the subtree $T_{e}$ (otherwise, $T_{e}$ would contain exactly one leaf $i \in I$, and then $\left.a_{e} \equiv a_{i} \not \equiv 0 \bmod m\right)$. Assume first that $v:=t(e)$ is not a leaf. From (15) and (16) we deduce the inequality

$$
\sum_{s\left(e^{\prime}\right)=v, e^{\prime} \neq \bar{e}}\left(\nu_{e^{\prime}}-1\right)=-1+\nu_{e} \leqslant 0 .
$$

Thus, we have $\nu_{e^{\prime}} \leqslant 1$ and $a_{e^{\prime}} \equiv 0 \bmod m$ for at least one positive edge $e^{\prime}$ with source $v$. Hence, after a finite number of steps, we find an edge $e$ such that $v:=t(e)$ is a leaf, $a_{e} \equiv 0 \bmod m$ and $\nu_{e} \leqslant 1$. This means that $\bar{X}_{v}$ is a new tail of $\bar{X}$ and that the conductor of the Artin-Schreier

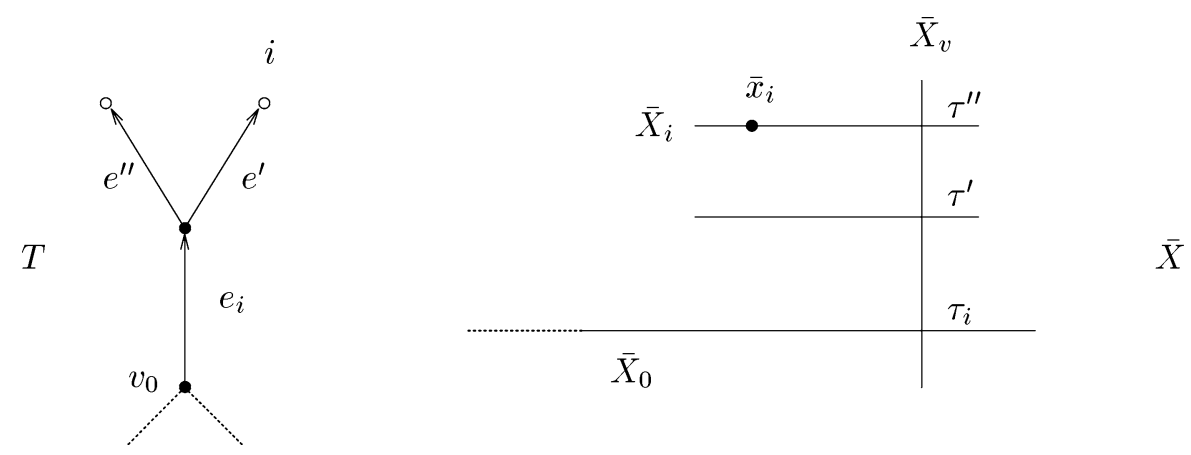

Fig. 1. 
cover $\bar{Y}_{v} \rightarrow \bar{Z}_{v}$ is $h_{v}=\nu_{e} \leqslant 1$. It follows that each connected component of $\bar{Y}_{v}$ is a tail of $\bar{Y}$ of genus 0 ; furthermore, no ramification point specializes to $\bar{Y}_{v}$. This contradicts the minimality of the stable model $Y_{R}$, and proves the second assertion of (i). The proof of the first assertion is similar, and uses the fact that the conductor of an Artin-Schreier cover is $\geqslant 1$.

Statement (ii) of the lemma follows immediately from (i). Indeed, a singular point on $\bar{X}_{0}$ which is not one of the $\tau_{i}$ would correspond to a positive edge $e$ with $a_{e} \equiv 0 \bmod m$ such that $\nu_{e}=1$ (by the assumption (29)).

To prove (iii), fix $i \in I$ and let us assume that $v:=t\left(e_{i}\right)$ is not a leaf. Let $E_{i}$ be the set of edges with source $v$ which are distinct from $\bar{e}_{i}$. There is a unique edge $e^{\prime} \in E_{i}$ such that $a_{e^{\prime}}=a_{i}$, and $a_{e} \equiv 0 \bmod m$ for all $e \in E_{i}-\left\{e^{\prime}\right\}$. In other words, the $m$-cyclic cover $\bar{Z}_{v} \rightarrow \bar{X}_{v} \cong \mathbb{P}_{k}^{1}$ is ramified at two points, so each component of $\bar{Z}_{v}$ has genus 0 . Since $\bar{Y}_{v} \rightarrow \bar{Z}_{v}$ is inseparable, each component of $\bar{Y}_{v}$ has genus 0 as well, and $\bar{Y}_{v}$ intersects with as many components of $\bar{Y}$ as $\bar{Z}_{v}$ intersect with components of $\bar{Z}$. Thus, the minimality of the stable model implies that $\left|E_{i}\right| \geqslant 2$. Since $\nu_{e_{i}}=\nu_{i} \in\{0,1\}$ (Eq. (33)) we have

$$
\sum_{e \in E_{i}}\left(\nu_{e}-1\right)=-1+\nu_{i} \in\{-1,0\}
$$

It follows from (i) that $\nu_{e^{\prime}} \geqslant 0$ and $\nu_{e}>1$ for all $e \in E_{i}-\left\{e^{\prime}\right\}$. We conclude that $E_{i}$ contains exactly two edges, $e^{\prime}$ and $e^{\prime \prime}$. Furthermore, we find $\nu_{i}=1, \nu_{e^{\prime}}=0$ and $\nu_{e^{\prime \prime}}=2$. The remaining assertion that $t\left(e^{\prime}\right)=i$ and that $t\left(e^{\prime \prime}\right)$ is a leaf is left to the reader (we will not use them in what follows).

Here is an immediate consequence of Lemma 3.3:

COROLlary 3.4. - Suppose that $f$ is not special. Then there exists an index $i \in I$ such that the following holds. Let $\bar{X}_{v}$ be the component which meets the original component $\bar{X}_{0}$ in $\tau_{i}$. Then $\bar{X}_{v}$ has nontrivial intersection with exactly three components of $\bar{X}$ (including $\bar{X}_{0}$ ). Furthermore, the torsor $Y \rightarrow Z$ has additive reduction over $\bar{X}_{v}$.

See Fig. 1 for an illustration of the relevant part of the tree $T$ and the curve $\bar{X}$, in the situation of Corollary 3.4.

\subsection{The proof of Theorem 3.2}

In Section 3.2 we have constructed, for any tuple of $\bar{K}$-rational points $\left(x_{i}\right)$ lifting $\left(\tau_{i}\right)$, a metacyclic cover $f: Y \rightarrow \mathbb{P}^{1}$ of type $\left(x_{i} ; a_{i}\right)$. In Section 3.3 we have analyzed the stable reduction of $f$. In this section we show that $f$ is special provided that $x_{i} \in \mathcal{D}_{i}$, with $\mathcal{D}_{i}$ as in (31), thus proving Theorem 3.2. We do this in two steps. First, we prove that $f$ is special if the $x_{i}$ are $K_{0}$-rational. Note that this is a special case of Theorem 3.2, as any $K_{0}$-rational point lifting $\tau_{i}$ is automatically a center of the disk $\mathcal{D}_{i}$. Then, we show that the cover $f$ remains special under a deformation which moves the branch point $x_{i}$ into an arbitrary point $x_{i}^{\prime} \in \mathcal{D}_{i}$.

Proposition 3.5. - Let $x_{1}, \ldots, x_{r}$ be $K_{0}$-rational points of $\mathbb{P}^{1}$ which lift $\tau_{1}, \ldots, \tau_{r}$. Then the metacyclic cover $f: Y \rightarrow \mathbb{P}^{1}$ of type $\left(x_{i} ; a_{i}\right)$ defined in Section 3.2 is special.

Proof. - By Proposition 1.3, the field of moduli of $f$ is $K^{\text {in }}=K_{0}\left(\zeta_{p}^{(m)}\right) \subset K_{0}\left(\zeta_{p}\right)$. We may assume that the field $K$ we have been working with in Sections 3.2 and 3.3 is the minimal extension of $K^{\text {in }}$ over which $f$ has stable reduction. It is proved in [13] that the extension $K / K^{\text {in }}$ is Galois, of degree prime-to- $p$. The Galois group $\Gamma:=\operatorname{Gal}\left(K / K^{\text {in }}\right)$ acts faithfully and $k$-linearly on $\bar{Y}$ (where $\bar{f}: \bar{Y} \rightarrow \bar{X}$ is the stable reduction of $f$ ), and this action commutes with 
the action of $H$. We obtain an induced action of $\Gamma$ on $\bar{Z}$ and $\bar{X}$. The action of $\Gamma$ on the original component $\bar{X}_{0}$ is trivial. Compare with Section 2.4.

In our setup, the $p$-cyclic cover $Y \rightarrow Z$ reduces to a $\boldsymbol{\mu}_{p}$-torsor $\bar{Y}_{0} \rightarrow \bar{Z}_{0}$ over the original component $\bar{X}_{0}$. Therefore, the field $K$ contains the $p$ th roots of unity. Let

$$
\widetilde{\Gamma}:=\operatorname{Gal}\left(K / K_{0}\left(\zeta_{p}\right)\right) \subset \Gamma .
$$

One shows easily that $\widetilde{\Gamma}$ acts trivially on $\bar{Y}_{0}$ (see also the proof of Theorem 2.10).

Now suppose that $f$ is not special. Let $\bar{X}_{v}$ be a component as in Corollary 3.4. In particular, $\bar{X}_{v}$ meets the rest of $\bar{X}$ in exactly three points. One of these points is $\tau_{i}$, where $\bar{X}_{v}$ meets $\bar{X}_{0}$. Let $\tau^{\prime}$ and $\tau^{\prime \prime}$ be the two other points, corresponding to the edges $e^{\prime}$ and $e^{\prime \prime}$ of Lemma 3.3(iii). Note that $\tau_{i}$ and $\tau^{\prime \prime}$ are the two branch points of the $m$-cyclic cover $\bar{Z}_{v} \rightarrow \bar{X}_{v}$. Since the action of $\widetilde{\Gamma}$ is trivial on $\bar{X}_{0}$ and commutes with the $\mathbb{Z} / m$-action on $\bar{Z}$, it fixes the point $\tau^{\prime}$ and the set $\left\{\tau_{i}, \tau^{\prime \prime}\right\}$. But the value of $\nu$ at the point $\tau_{i}$ and $\tau^{\prime \prime}$ is different, so $\tau_{i}$ and $\tau^{\prime \prime}$ are fixed individually. Therefore, $\widetilde{\Gamma}$ acts trivially on $\bar{X}_{v}$.

Choose a point $\bar{y} \in \bar{Y}$ above $\tau_{i}$ and let $\bar{z}$ be the image of $\bar{y}$ in $\bar{Z}$. Note that $\bar{y}$ and $\bar{z}$ are ordinary double points of $\bar{Y}$ and $\bar{Z}$, respectively. Let $\widehat{Y}_{R}$ be the completion of $Y_{R}$ at $\bar{y}$ and $\widehat{Z}_{R}$ the completion of $Z_{R}$ at $\bar{z}$. Since $Y_{R}$ is semistable, $\widehat{Y}_{R}$ is a formal annulus, of the form $\widehat{Y}_{R} \cong \operatorname{Spec} R[[u, v \mid u v=\pi]]$, with $\pi \in R$. Corollary 3.4 states that we have additive reduction over the component $\bar{Z}_{v}$. By Remark 2.7, this implies

$$
0<v_{R}(\pi)<\frac{1}{(p-1) h_{i}}
$$

Let $\lambda:=\zeta_{p}-1$. Note that $v_{R}(\lambda)=1 /(p-1)$ and that $\lambda$ is a uniformizer of $K_{0}\left(\zeta_{p}\right)$. We conclude from (36) that $K_{0}\left(\zeta_{p}, \lambda^{1 / N}\right) \subset K$ and $v_{R}(\pi)=c /(p-1) N$, with $N>h_{i}>m$ and $c$ prime-to- $N$. Moreover, $N$ is prime-to- $p$ because $K / K_{0}$ is of degree prime-to- $p$. Let $\zeta_{N}$ be a primitive $N$ th root of unity and $\sigma \in \widetilde{\Gamma}$ such that $\sigma\left(\lambda^{1 / N}\right)=\zeta_{N} \lambda^{1 / N}$. Then $\sigma(\pi)=\zeta_{N}^{c} \pi$. Up to permutation, the parameter $u$ (resp. $v$ ) of the formal annulus $\widehat{Y}_{R}$ reduces to a local parameter of $\bar{Y}_{0}$ (resp. $\bar{Y}_{v}$ ) at $\bar{y}$. Applying $\sigma$ to the equation $u v=\pi$ and using the fact that $\widetilde{\Gamma}$ acts trivially on $\bar{Y}_{0}$, we find that $\sigma$ induces an automorphism of $\bar{Y}_{v}$ of order $N$ which fixes $\bar{y}$. We conclude that $\sigma$ induces an automorphism of $\bar{X}_{v}$ of order at least $N /(N, m)>1$. But this contradicts the fact (proved earlier) that $\widetilde{\Gamma}$ acts trivially on $\bar{X}_{v}$. The proposition follows.

Let $f: Y \rightarrow \mathbb{P}^{1}$ be as in Proposition 3.5. In particular, $f$ is special. Proposition 3.6 below states that, given points $x_{i}^{\prime} \in \mathcal{D}_{i}$, we can deform $f$ into a cover $f^{\prime}$ of type $\left(x_{i}^{\prime} ; a_{i}\right)$. By construction, the cover $f^{\prime}$ is special and gives rise to $\left(\tau_{i} ; a_{i} ; \nu_{i} ; \omega_{0}\right)$. This completes the proof of Theorem 3.2.

Proposition 3.6. - For $i \in I$, choose $x_{i}^{\prime} \in \mathcal{D}_{i}$. There exists a special cover $f^{\prime}: Y^{\prime} \rightarrow \mathbb{P}^{1}$ of type $\left(x_{i}^{\prime} ; a_{i}\right)$ which gives rise to the special degeneration datum $\left(\tau_{i} ; a_{i} ; \nu_{i} ; \omega_{0}\right)$.

Proof. - By Theorem 2.10, the disk $\mathcal{D}_{i}$ (as defined by (31), with center $x_{i}=\tilde{x}_{i}$ ) corresponds to the tail $\bar{X}_{i}$, i.e.

$$
\mathcal{D}_{i}=\left\{x \in \mathbb{P}^{1}(\bar{K}) \mid x \text { specializes to a point on } \bar{X}_{i}-\left\{\tau_{i}\right\} \cong \mathbb{A}_{k}^{1}\right\} .
$$

In particular, $x_{i}^{\prime}$ specializes to a point $\bar{x}_{i}^{\prime} \in \bar{X}_{i}-\left\{\tau_{i}\right\}$.

We define a finite, $H$-invariant map $\bar{f}^{\prime}: \bar{Y}^{\prime} \rightarrow \bar{X}$ between semistable $k$-curves. It is determined, up to unique isomorphism, by the following requirements (compare with [13], §3.2). Over the original component $\bar{X}_{0}$, the maps $\bar{f}: \bar{Y} \rightarrow \bar{X}$ and $\bar{f}^{\prime}: \bar{Y}^{\prime} \rightarrow \bar{X}$ are the same. Even more, 
there exists an étale map $U \rightarrow \bar{X}$ whose image contains $\bar{X}_{0}$ such that the pullbacks $\left.\bar{f}\right|_{U}$ and $\left.\bar{f}^{\prime}\right|_{U}$ are isomorphic, as $m$-cyclic covers of $U$. Finally, for each $i \in I$, the restriction of $\bar{f}^{\prime}$ to the open subset $\bar{X}_{i}-\left\{\tau_{i}\right\} \subset \bar{X}$ is a (possibly disconnected) $H$-cover which is at most tamely ramified at $\bar{x}_{i}^{\prime}$.

Arguing as in [13], §3.2, one shows that $\bar{f}^{\prime}: \bar{Y}^{\prime} \rightarrow \bar{X}$ lifts to a finite, $H$-invariant morphism $f_{R}^{\prime}: Y_{R}^{\prime} \rightarrow X_{R}$ between semistable $R$-curves such that the following holds. First, $f_{R}^{\prime}$ is the stable model of an $H$-cover $f^{\prime}: Y^{\prime} \rightarrow \mathbb{P}^{1}$ of type $\left(x_{i}^{\prime} ; a_{i}\right)$. Second, the restrictions of $f_{R}^{\prime}$ and $f_{R}$ to the formal completion $\mathcal{X}_{0}$ of $X_{R}$ along $\bar{X}_{0}$ are isomorphic (as finite, $H$-invariant maps to $\mathcal{X}_{0}$ ). It follows immediately that the $H$-cover $f^{\prime}$ is special and gives rise to the special degeneration datum $\left(\tau_{i} ; a_{i} ; \nu_{i} ; \omega_{0}\right)$. This proves Proposition 3.6 and therefore completes the proof of Theorem 3.2.

\subsection{The case $r=4$}

Let us call two special degeneration data $\left(\tau_{i} ; a_{i} ; \nu_{i} ; \omega_{0}\right)$ and $\left(\tau_{i}^{\prime} ; a_{i}^{\prime} ; \nu_{i}^{\prime} ; \omega_{0}^{\prime}\right)$ equivalent if there exists a $\mathbb{Z} / m$-equivariant isomorphism $\phi: \bar{Z}_{0} \stackrel{\sim}{\rightarrow} \bar{Z}_{0}^{\prime}$ (where $\bar{Z}_{0} \rightarrow \mathbb{P}_{k}^{1}$ and $\bar{Z}_{0}^{\prime} \rightarrow \mathbb{P}_{k}^{1}$ are the corresponding $m$-cyclic covers) such that $\phi^{*} \omega_{0}^{\prime}=c \omega_{0}$, for some constant $c \in \mathbb{F}_{p}^{\times}$. In this section we determine all special degeneration data $\left(\tau_{i} ; a_{i} ; \nu_{i} ; \omega_{0}\right)$ with four branch points, up to equivalence.

Fix $p, m$ and $\left(a_{i}\right)$, as in the beginning of Section 1.1. It is clear that for given prime $p$, there is only a finite number of possibilities for $m$ and $\left(a_{i}\right)$, which are easy to describe. After reordering the indices, we may assume that $\nu_{1}=1$ and $\nu_{2}=\nu_{3}=\nu_{4}=0$. Furthermore, after a projective linear transformation, we may assume that $\tau_{1}=0, \tau_{2}=1, \tau_{4}=\infty$. We write $\lambda$ instead of $\tau_{3}$; note that $\lambda \in k-\{0,1\}$. Let $Z_{\lambda} \rightarrow \mathbb{P}^{1}$ be the $m$-cyclic cover given by the equation

$$
z^{m}=x^{a_{1}}(x-1)^{a_{2}}(x-\lambda)^{a_{3}} .
$$

By an easy calculation, involving the divisors of $z, x$ and $\mathrm{d} x$, one shows that any differential $\omega_{0}$ on $Z_{\lambda}$ satisfying Eq. (29) is of the form

$$
\omega_{0}=\mu \frac{z \mathrm{~d} x}{(x-1)(x-\lambda)}=\mu \frac{x \mathrm{~d} x}{w}
$$

for some constant $\mu \in k^{\times}$. Here we have set $w:=z^{-1} x(x-1)(x-\lambda)$. Note that

$$
w^{m}=x^{a_{1}^{\prime}}(x-1)^{a_{2}^{\prime}}(x-\lambda)^{a_{3}^{\prime}}
$$

with $a_{i}^{\prime}:=m-a_{i}$.

Recall that the Cartier operator is defined as the unique additive map $\mathcal{C}$ on differentials such that

$$
\begin{aligned}
\mathcal{C}\left(u^{p} \omega\right) & =u \mathcal{C}(\omega), \\
\mathcal{C}(\mathrm{d} u) & =0, \quad \text { and } \\
\mathcal{C}(\mathrm{d} u / u) & =\mathrm{d} u / u,
\end{aligned}
$$

for all rational functions $u$ and differentials $\omega$ on $Z_{\lambda}$. We want to find all $\lambda$ and $\mu$ such that $\mathcal{C}\left(\omega_{0}\right)=\omega_{0}$. We set $\alpha:=(p-1) / m$ and and $f(x):=w^{m}=x^{a_{1}^{\prime}}(x-1)^{a_{2}^{\prime}}(x-\lambda)^{a_{3}^{\prime}}$. Following [22] and using (40)-(42), we compute

$4^{\mathrm{e}}$ SÉRIE - TOME $36-2003-\mathrm{N}^{\circ} 1$ 


$$
\begin{aligned}
\mathcal{C}\left(\omega_{0}\right) & =\mu^{1 / p} \mathcal{C}\left(\frac{x f(x)^{\alpha} \mathrm{d} x}{w^{p}}\right)=\mu^{1 / p} w^{-1} \sum_{k=\alpha a_{1}^{\prime}}^{\alpha\left(2 m-a_{1}\right)} c_{k}^{1 / p} \mathcal{C}\left(x^{k+1} \mathrm{~d} x\right) \\
& =\mu^{1 / p}\left(c_{p-2}^{1 / p}+c_{2 p-2}^{1 / p} x\right) \frac{\mathrm{d} x}{w},
\end{aligned}
$$

where $c_{k}$ is the coefficient of $x^{k}$ in $f(x)^{\alpha}$. We see that $\mathcal{C}\left(\omega_{0}\right)=\omega_{0}$ implies $c_{p-2}=0$. On the other hand, if $c_{p-2}=0$, then $c_{2 p-2} \neq 0$, because $\mathcal{C}\left(\omega_{0}\right) \neq 0$, by Lemma 1.4(iv). Therefore, if $c_{p-2}=0$, we can set $\mu:=c_{2 p-2}^{1 /(p-1)}$, to obtain $\mathcal{C}\left(\omega_{0}\right)=\omega_{0}$. We conclude: if $\lambda$ is a zero of

$$
\Phi(\lambda)=\sum_{l=0}^{N}\left(\begin{array}{c}
\alpha a_{2}^{\prime} \\
N-l
\end{array}\right)\left(\begin{array}{c}
\alpha a_{3}^{\prime} \\
l
\end{array}\right) \lambda^{l}
$$

(with $N:=p-\alpha a_{1}^{\prime}-2=\alpha a_{1}-1$ ), then there exists a differential $\omega_{0}$ on $Z_{\lambda}$ satisfying (29) and (30), unique up to a constant factor in $\mathbb{F}_{p}^{\times}$.

LEMMA 3.7. - The zeros of the polynomial $\Phi$ in (44) are simple and $\neq 0,1$.

Proof. - It is shown in [19] that the zeros of $\Phi$ other than 0 and 1 are simple (the reason is that $\Phi$ satisfies a certain hypergeometric differential equation). Hence we have to show that $\Phi(0), \Phi(1) \neq 0$. Recall that $a_{i}^{\prime}=m-a_{i}$ and $\sum_{i} a_{i}=m$. Since $a_{2}^{\prime}=a_{1}+2 m-a_{3}^{\prime}-a_{4}^{\prime} \geqslant a_{1}$, we have $\alpha a_{2}^{\prime}>\alpha a_{1}-1=N$, so $\Phi(0)=\left(\begin{array}{c}\alpha a_{2}^{\prime} \\ N\end{array}\right) \neq 0$. On the other hand, we have

$$
\Phi(1)=\text { coeff. of } x^{N} \text { in }(x-1)^{\alpha\left(a_{2}^{\prime}+a_{3}^{\prime}\right)}=\left(\begin{array}{c}
\alpha\left(a_{2}^{\prime}+a_{3}^{\prime}\right) \\
N
\end{array}\right) .
$$

But $\alpha\left(a_{2}^{\prime}+a_{3}^{\prime}\right)-N=\alpha\left(a_{1}^{\prime}+a_{2}^{\prime}+a_{3}^{\prime}-m\right)+1=\alpha\left(m+a_{4}\right)+1>1$, so $\Phi(1) \neq 0$ as well.

We can summarize the discussion as follows:

Proposition 3.8. - Let $r:=4$. Given $p, m,\left(a_{i}\right)$ and $\left(\nu_{i}\right)$ (satisfying the usual conditions, and with $\left.\nu_{1}=1\right)$, there are exactly $\alpha a_{1}-1$ nonequivalent special degeneration data $\left(\tau_{i} ; a_{i} ; \nu_{i} ; \omega_{0}\right)$. In particular, for given $p$, there exist only a finite number of nonequivalent special degeneration data, and they are all defined over some finite field $\mathbb{F}_{p^{n}}$. Therefore, we may assume $k=\overline{\mathbb{F}}_{p}$ throughout.

Remark 3.9. - For $r>4$, it is still true that there exist only a finite number of equivalence classes of special degeneration data, for fixed $p$. This is less obvious than for $r=4$, because the polynomial $\Phi$ is replaced by a system of $r-3$ equations in $r-3$ variables. However, a deformation argument shows that this system has only finitely many solutions, see [21]. It would be interesting to obtain a formula for the number of solutions, as in the case $r=4$.

\section{Acknowledgements}

The author would like to thank Professor Raynaud for useful comments on an earlier version of this paper. He also gratefully acknowledges financial support from the Deutsche Forschungsgemeinschaft.

\section{REFERENCES}

[1] Bosch S., LÜtкebohmert W., Raynaud M., Néron Models, in: Ergebnisse der Mathematik und ihrer Grenzgebiete, Vol. 21, Springer-Verlag, 1990. 
[2] Bouw I.I., Pries R.J., Rigidity, reduction, and ramification, Math. Ann., To appear.

[3] Bouw I.I, Wewers S., Reduction of covers and Hurwitz spaces, Available at math.AG/0005120, 2000.

[4] Dèbes P., Douai J.-C., Algebraic covers: Field of moduli versus field of definition, Ann. Scient. Ecole Norm. Sup. 30 (1997) 303-338.

[5] Gille P., Le groupe fondamental sauvage d'une courbe affine, in: Bost J.-B., Loeser F., Raynaud M. (Eds.), Courbe semi-stable et groupe fondamental en géométrie algébrique, in: Progress in Math., Vol. 187, Birkhäuser, 2000, pp. 217-230.

[6] Green B., Matignon M., Order $p$ automorphisms of the open disc of a $p$-adic field, J. AMS 12 (1) (1999) 269-303.

[7] HARris J., MumFord D., On the Kodaira dimension of the moduli space of curves, Invent. Math. 67 (1982) 23-86.

[8] Henrio Y., Arbres de Hurwitz et automorphismes d'ordre p des disques et des couronnes p-adiques formels, PhD thesis, Bordeaux, 1999.

[9] KnUdsen F.F., The projectivity of the moduli space of stable curves, II, Math. Scand. 52 (1983) 161-199.

[10] Milne J., Étale Cohomology, Princeton Univ. Press, 1980.

[11] Mumford D., Abelian Varieties, Oxford Univ. Press, 1970.

[12] RaYnAUD M., p-groupes et réduction semi-stable des courbes, in: Cartier P. (Ed.), Grothendieck Festschrift III, in: Progress in Math., Vol. 88, Birkhäuser, 1990, pp. 179-197.

[13] RaYnAUd M., Spécialisation des revêtements en caractéristique $p>0$, Ann. Scient. Ecole Norm. Sup. (1999) 87-126.

[14] SAÏDI M., Galois covers of degree $p$ and semistable reduction of curves, Personal notes.

[15] SAÏDi M., Revêtements modérés et groupe fondamental de graphe de groupes, Comp. Math. 107 (1997) 321-340.

[16] SERrE J-P., Sur la topologie des variétés algébriques en caractéristique p, in: Symp. Int. Top. Alg., 1958, pp. 24-53.

[17] Serre J-P., Corps Locaux, Hermann, 1968.

[18] SERrE J-P., Topics in Galois Theory, in: Research Notes in Mathematics, Vol. 1, Jones and Bartlett Publishers, 1992, Lecture notes prepared by Henri Darmon.

[19] Stienstra J., VAn Der Put M., VAn Der Marel B., On p-adic monodromy, Math. Z. 208 (1991) 309-325.

[20] WEWERS S., Three point covers with bad reduction, In preparation.

[21] WEWERS S., Deformation of curves with group scheme action, Preprint, 2001.

[22] YUi N., On the Jacobian variety of the Fermat curve, J. Algebra 65 (1980) 1-35.

(Manuscrit reçu le 9 mai 2001; accepté, après révision, le 7 janvier 2002.)

\footnotetext{
Stefan WEWERS

Mathematisches Institut,

Universität Bonn,

Beringstraße 4,

53115 Bonn, Germany

E-mail: wewers@math.uni-bonn.de
} 